\title{
Shared Control Design Methodologies of an Electric Wheelchair for Individuals with Severe Disabilities using Reinforcement Learning
}

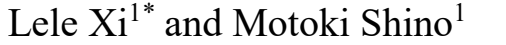

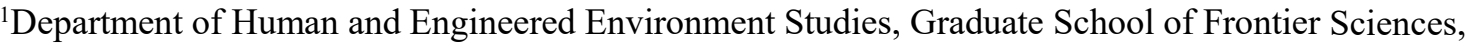

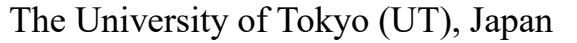

xi.lele@atl.k.u-tokyo.ac.jp

Received: August 19, 2019; Accepted: October 10, 2020; Published: October 17, 2020

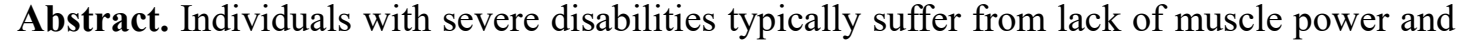

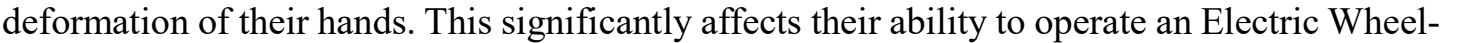

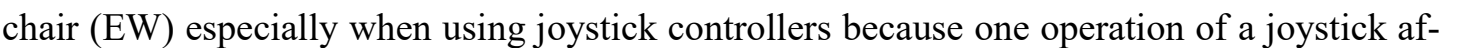

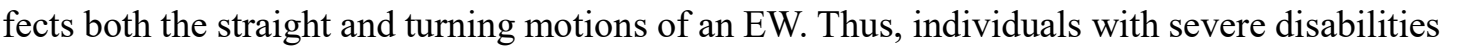

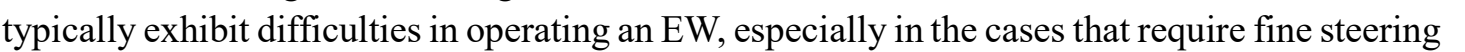

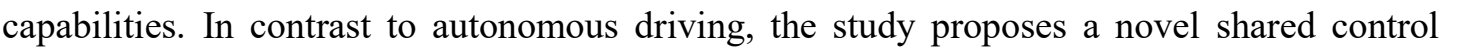

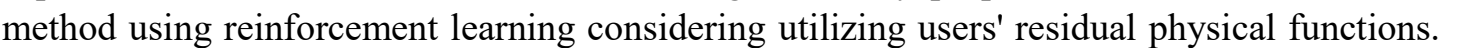

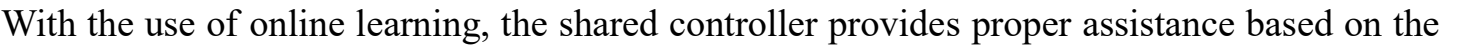

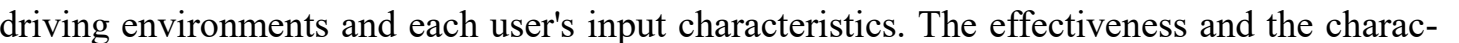

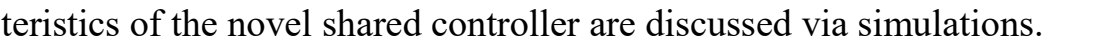

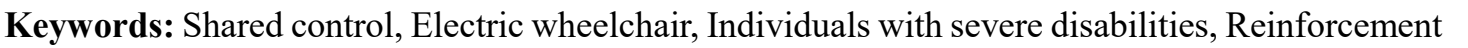

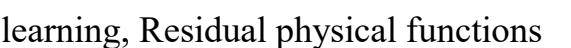

\section{Introduction}

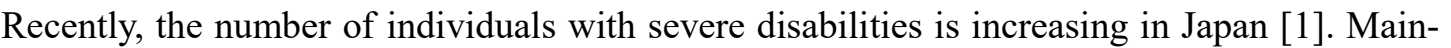

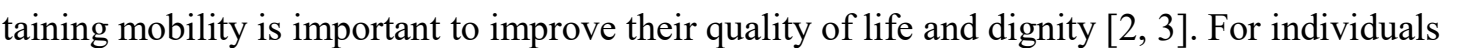

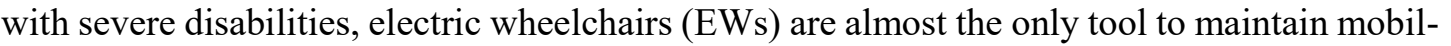

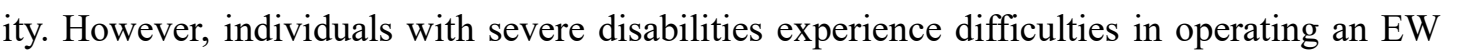

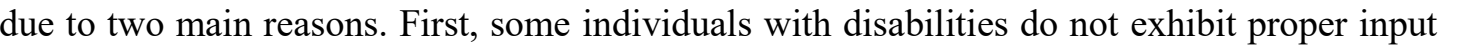

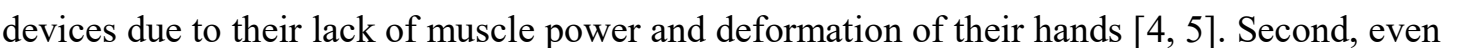

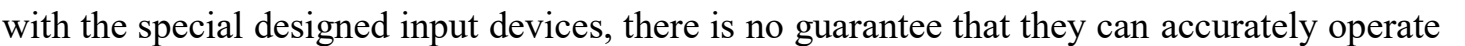

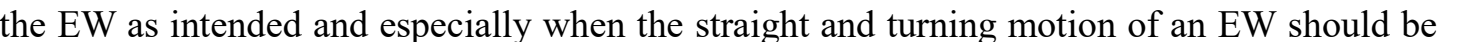




\section{Journal of Advanced Simulation in Science and Engineering}

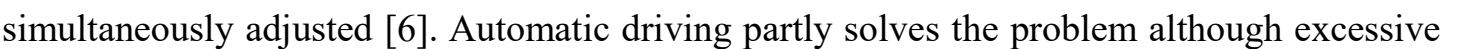

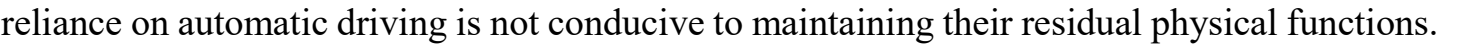

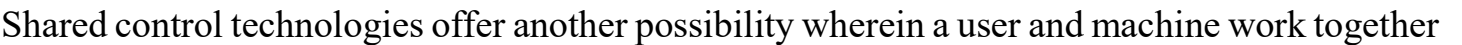

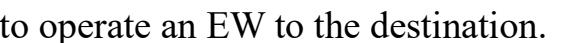

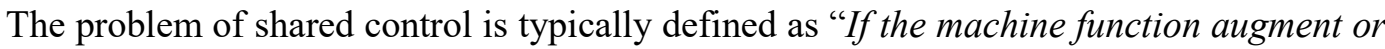
replace one or more of the human senses, the user must be able to reach his goal and also understand what the machine is doing. This two-way interaction must exist in any shared con-

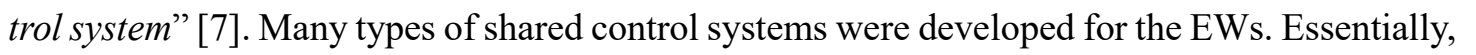

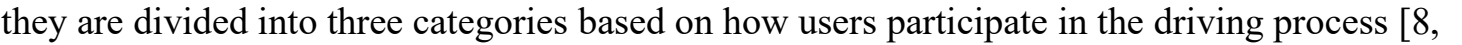

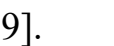

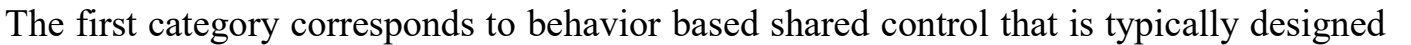

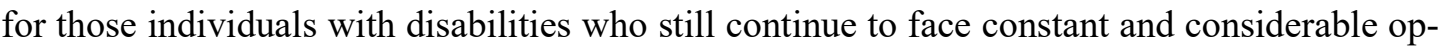

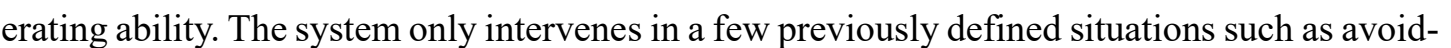

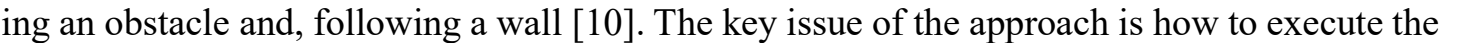

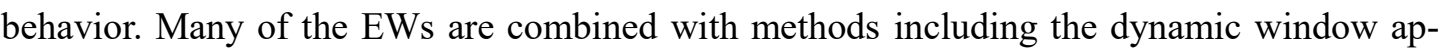

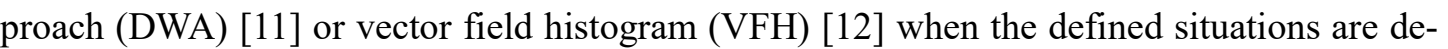

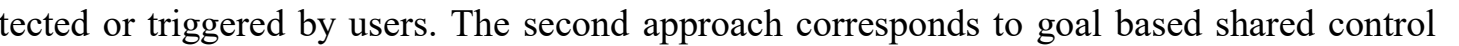
ए ए।

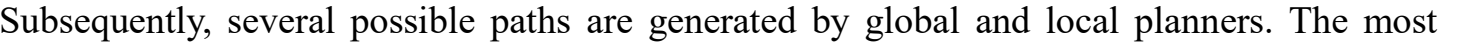

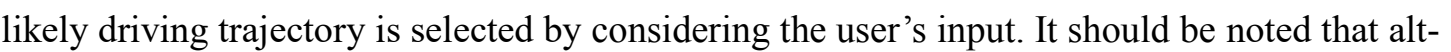

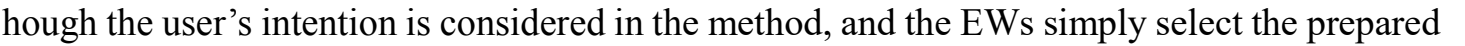

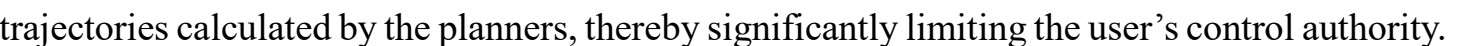

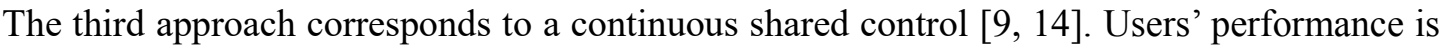

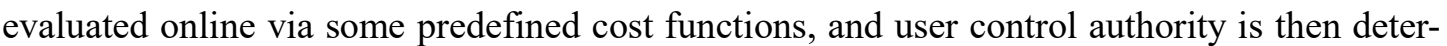

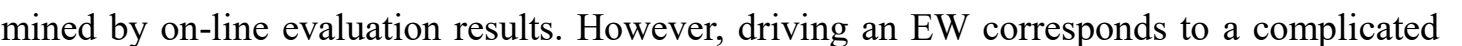

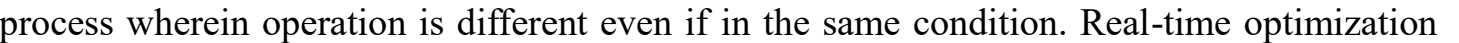
limits users' performance, and the construction of the cost functions is always a difficult $\square \square \square$ पाणा

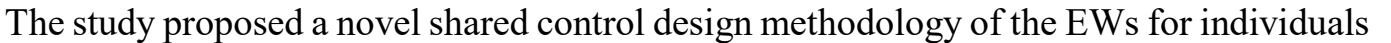

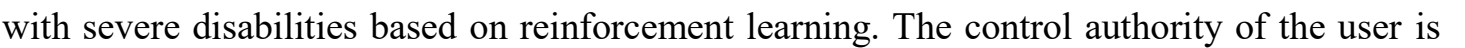

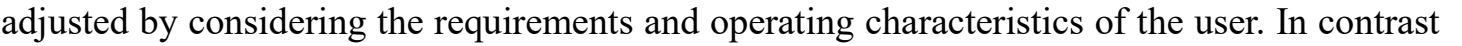

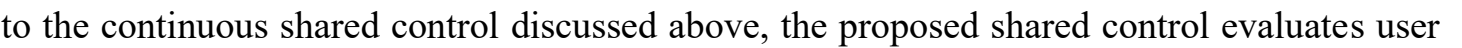

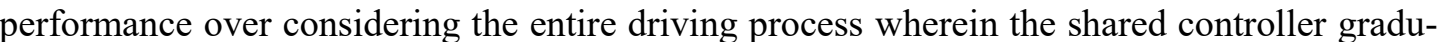

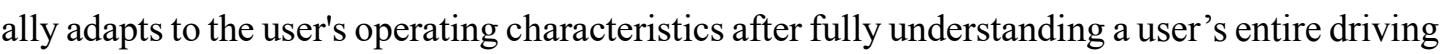

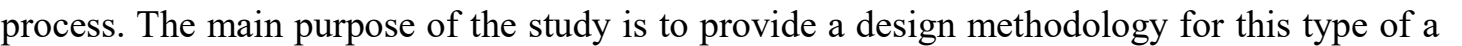

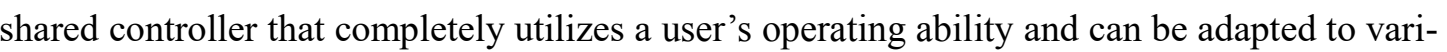

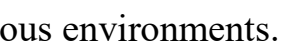

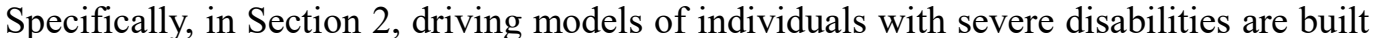


Journal of Advanced Simulation in Science and Engineering

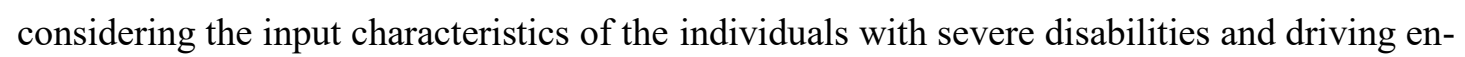

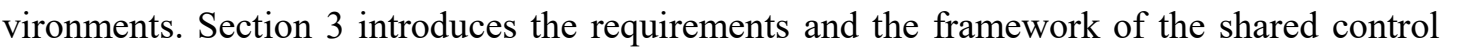

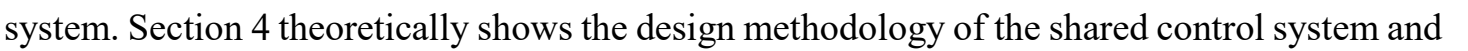

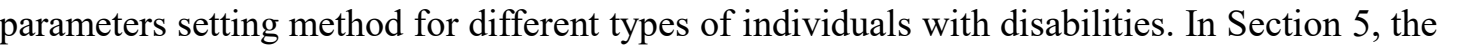

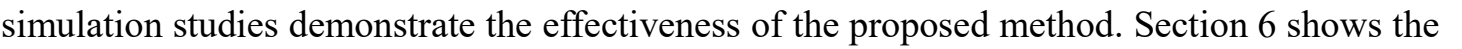

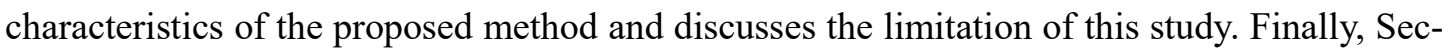

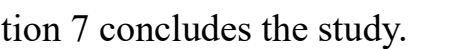

\section{Driving environments and models for individuals with severe dis- abilities}

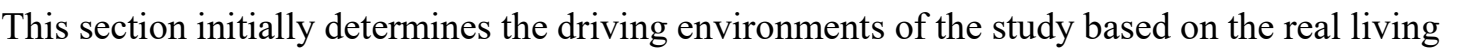

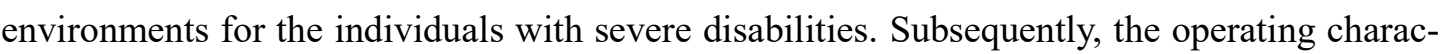

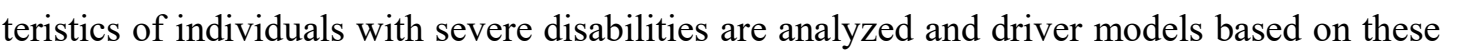

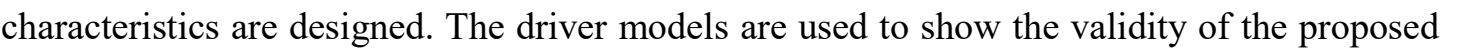

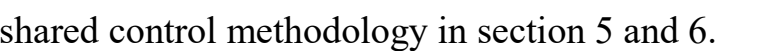

\subsection{Driving environments for individuals with severe disabilities}

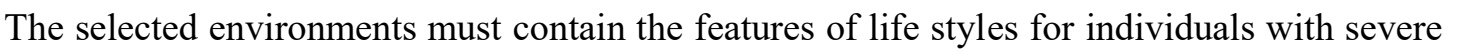

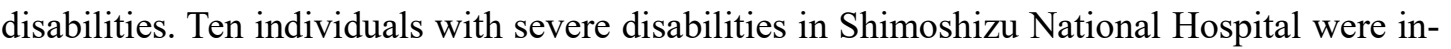

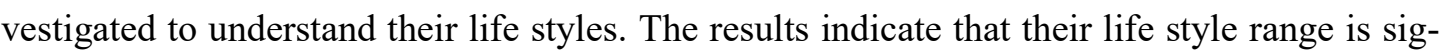

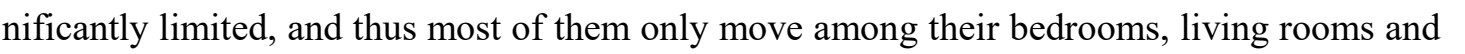

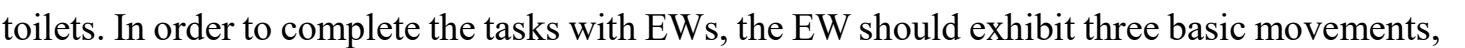

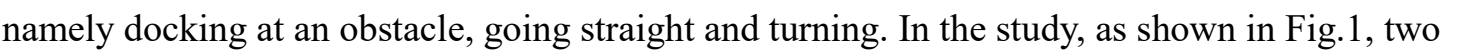

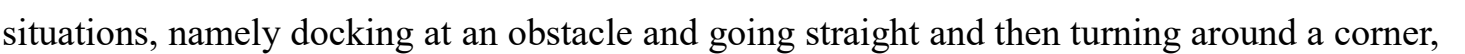

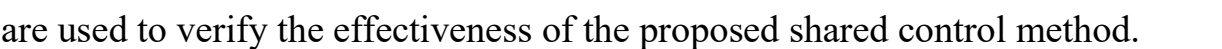
$\square$
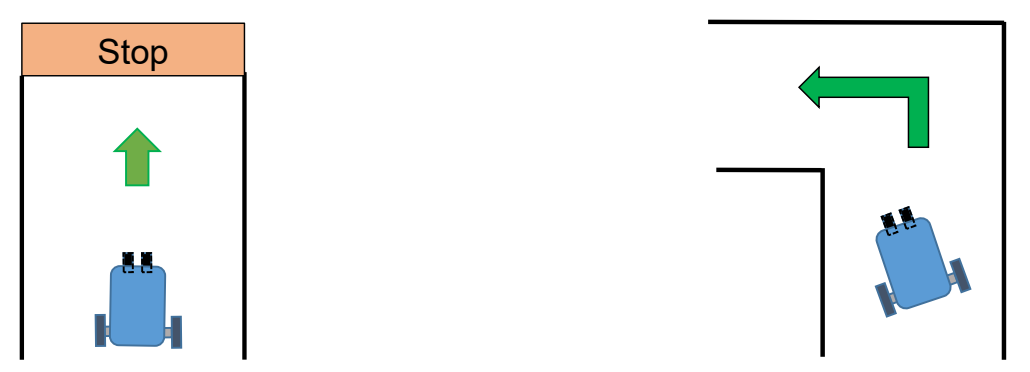

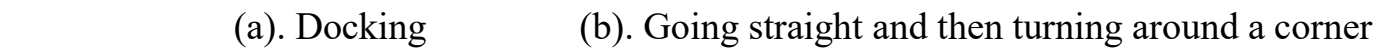




\section{Journal of Advanced Simulation in Science and Engineering}

\subsection{Operating characteristics of individuals with severe disabilities}

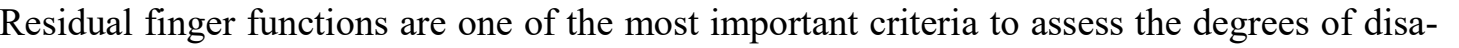

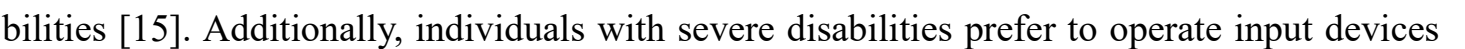

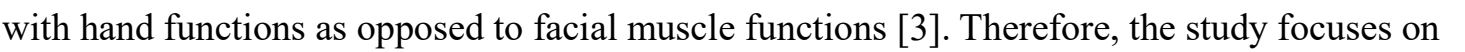

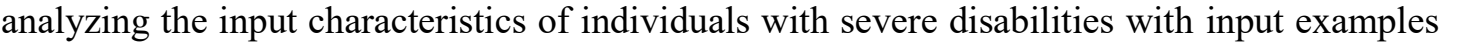

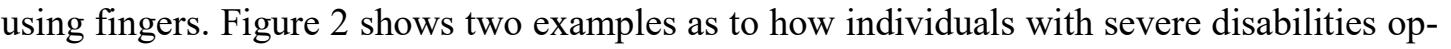

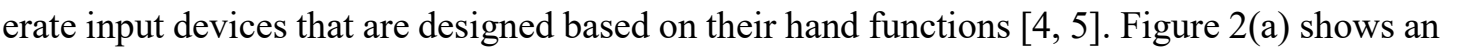

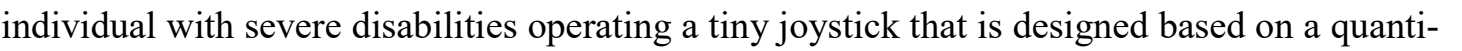

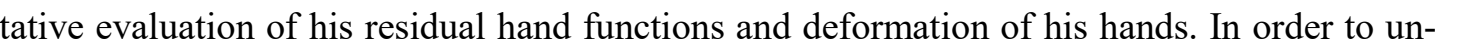

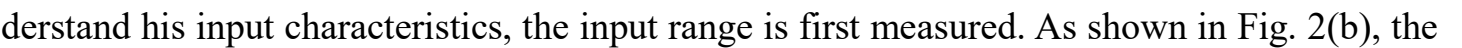

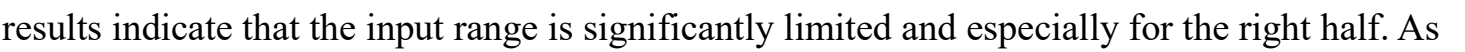

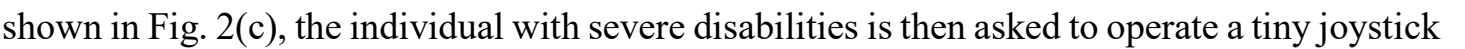

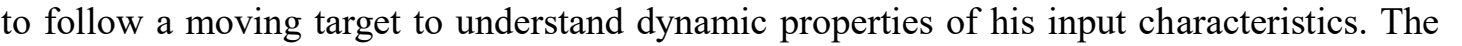

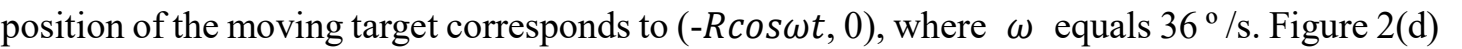

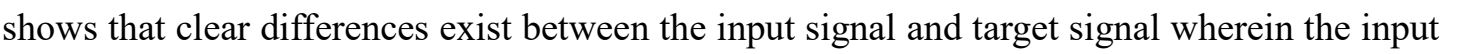

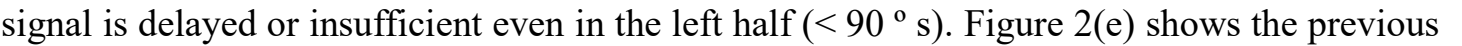

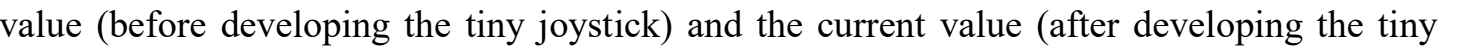

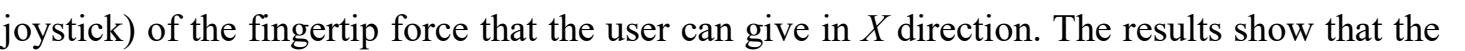

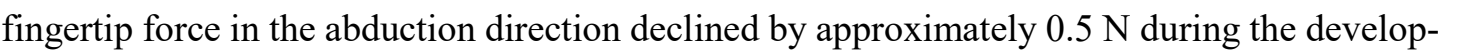

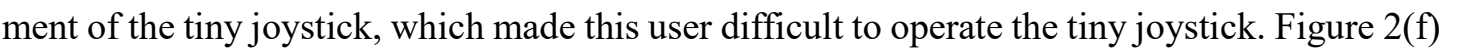

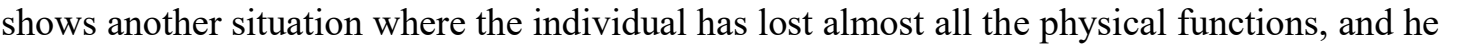

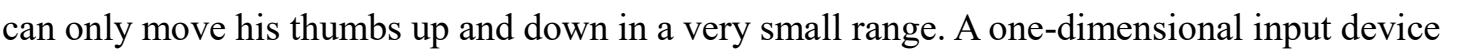

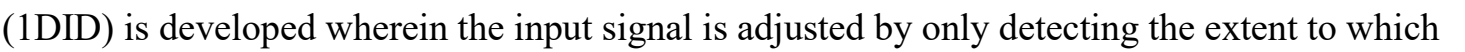
ए ए

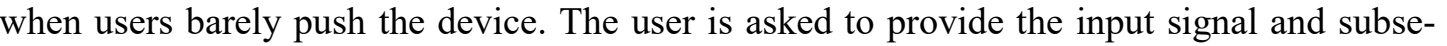

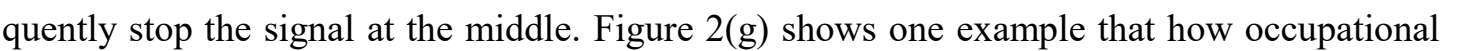

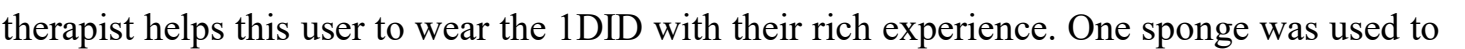

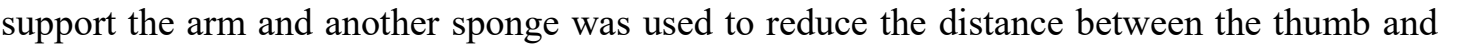

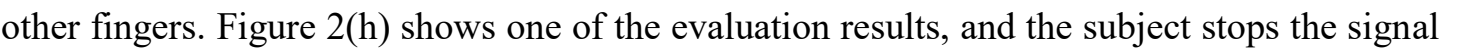

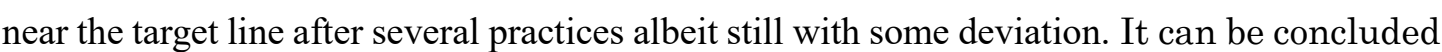
that although 1DIDs are used to express an individual's intention to a certain extent, it is al-

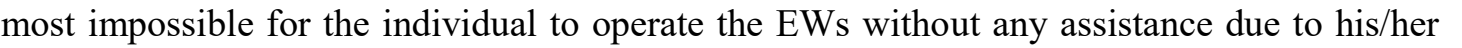

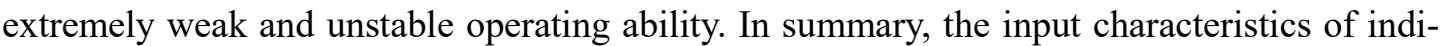

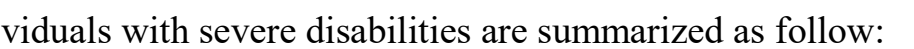

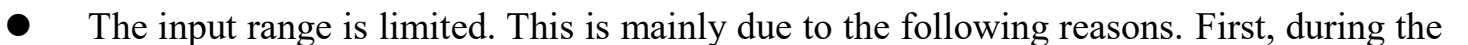


Journal of Advanced Simulation in Science and Engineering

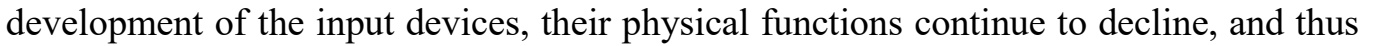

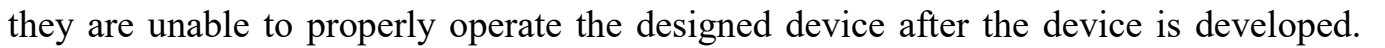

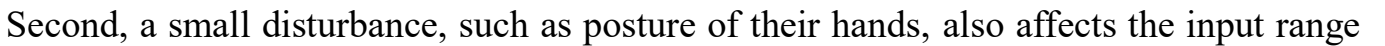

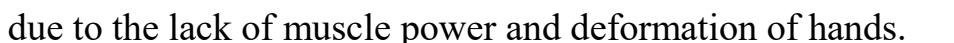

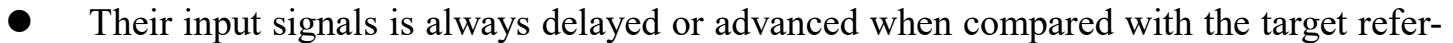

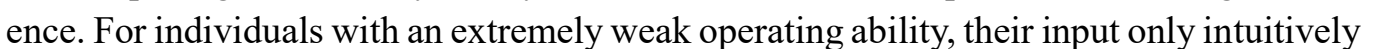
represents the subjects' intention although driving an EW in real environments corre-

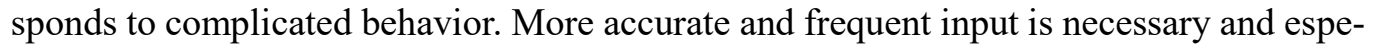

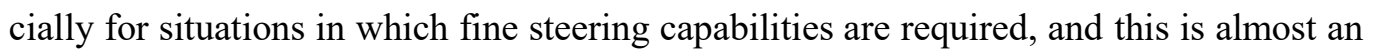

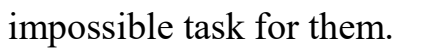
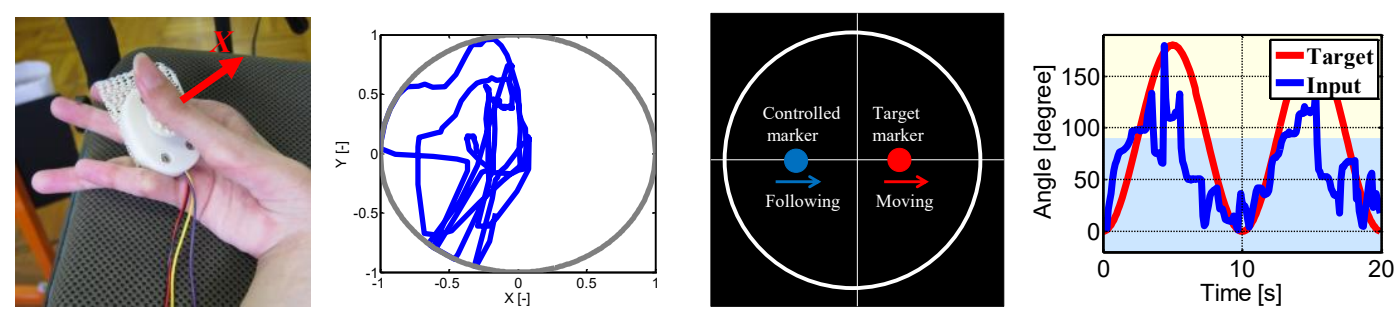

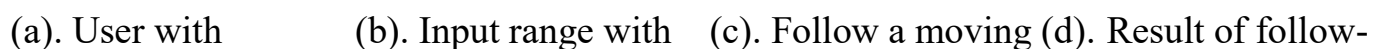

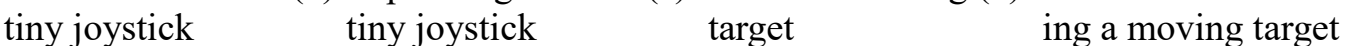
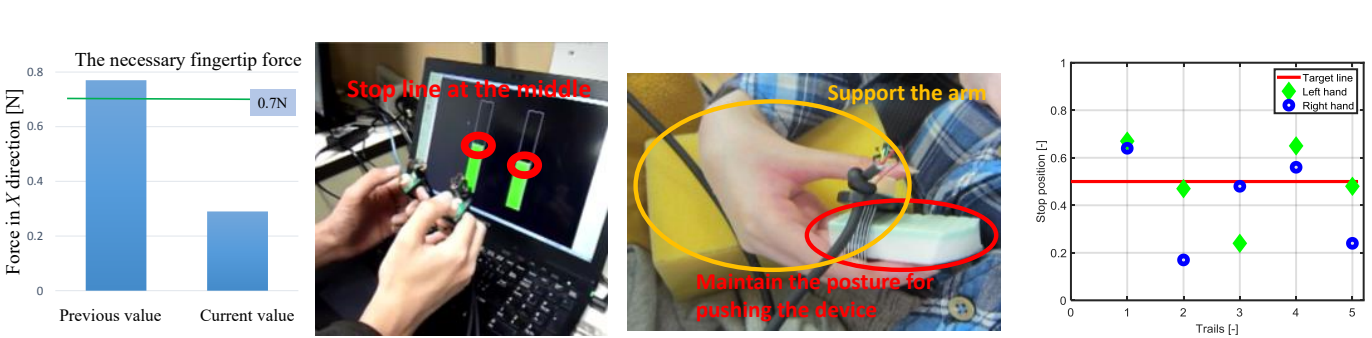

$\square$

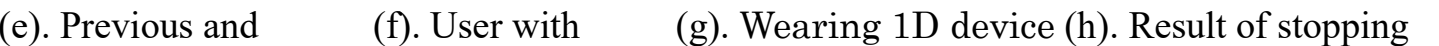

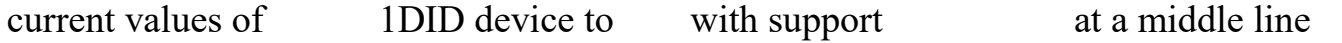

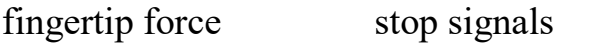

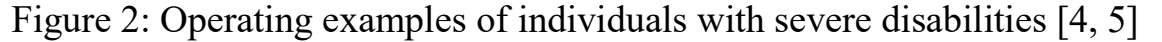

\subsection{Driving models for individuals with disabilities}

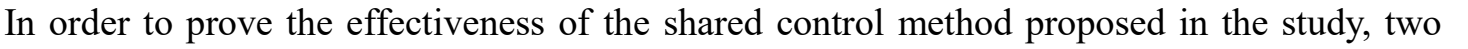

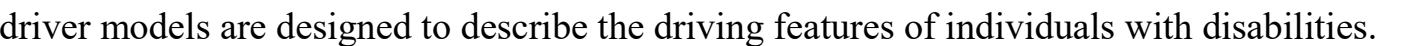

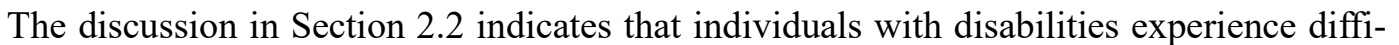

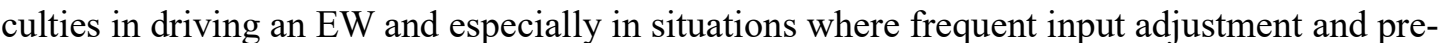

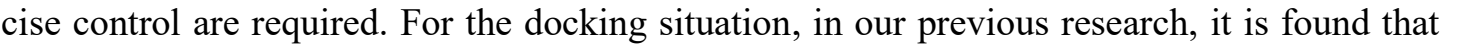

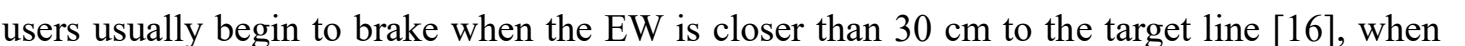




\section{Journal of Advanced Simulation in Science and Engineering}

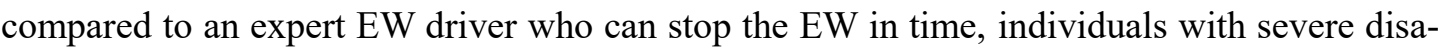

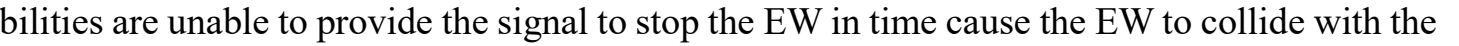

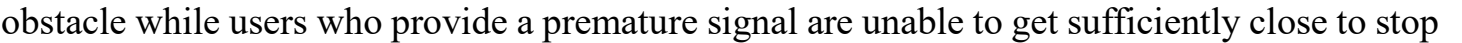

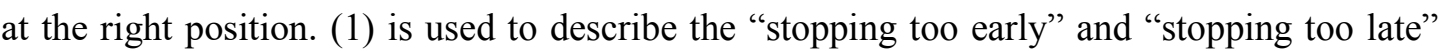

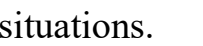

$$
v=\left\{\begin{array}{cc}
v_{o}, & \Delta y>y_{\text {brake }} \\
v_{0}-k\left(t-t_{\text {brake }}\right), & \Delta y<y_{\text {brake }}, v_{0}>k\left(t-t_{\text {brake }}\right) \\
0, & v_{0}<k\left(t-t_{\text {brake }}\right)
\end{array}\right.
$$

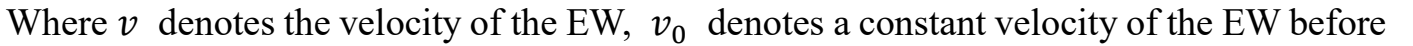

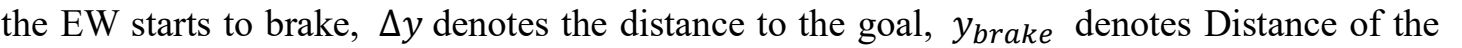

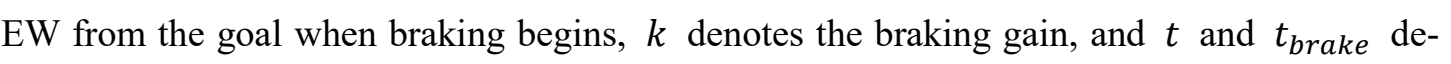

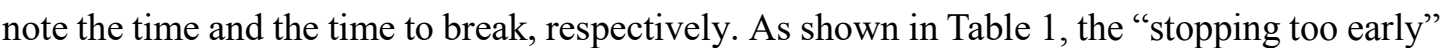

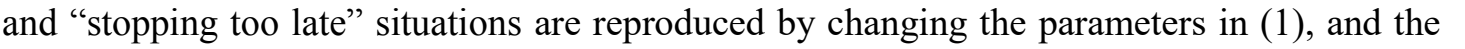

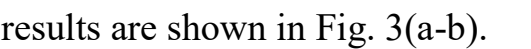

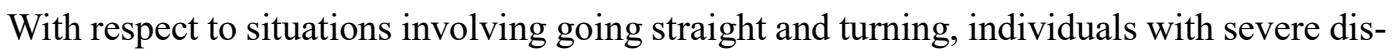

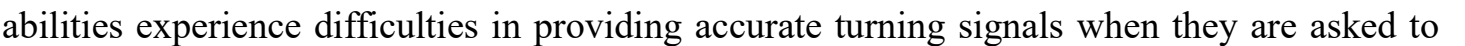

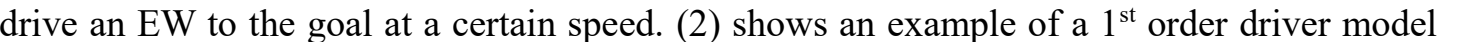
एणाणाए

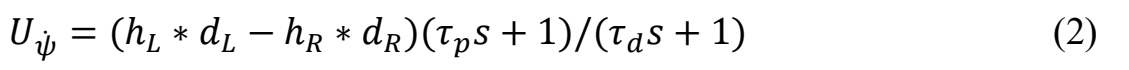

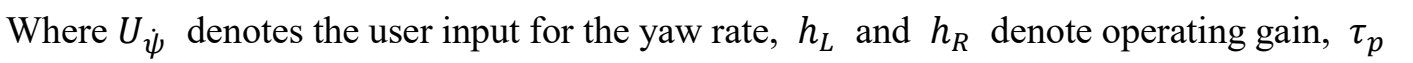

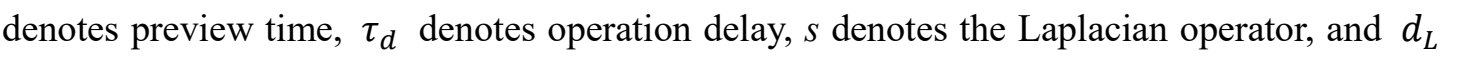

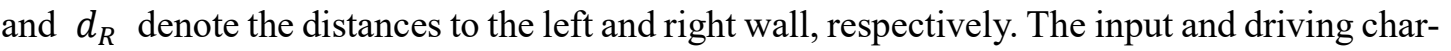

\begin{tabular}{|c|c|c|c|c|}
\hline 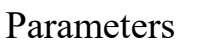 & 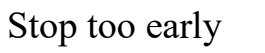 & 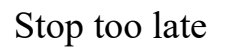 & 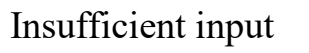 & 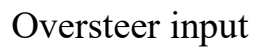 \\
\hline$v_{0}$ & पणाण एण & पण口冋 एव & $\square$ & $\square$ \\
\hline$y_{\text {brake }}$ & पण口冋 & $\square$ पाण & $\square$ & $\square$ \\
\hline$k \square$ & $\square$ & $\square \square$ & $\square$ & $\square$ \\
\hline$h_{L}$ & $\square$ & 四 & $\square \square$ & $\square \square$ \\
\hline$h_{R}$ & $\square$ & 四 & $\square \mathrm{ll}$ & $\square \square$ \\
\hline$\tau_{p}$ & $\square$ & $\square$ & $\square$ & $\square$ \\
\hline$\tau_{d}$ & $\square$ & 四 & $\square \mathrm{ll}$ & $\square$ \\
\hline
\end{tabular}

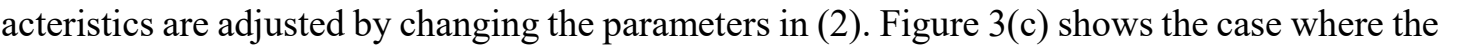

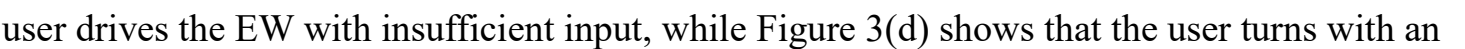

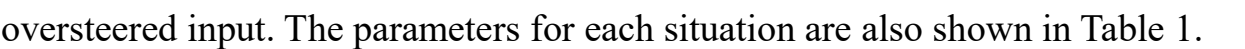

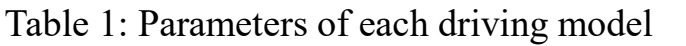




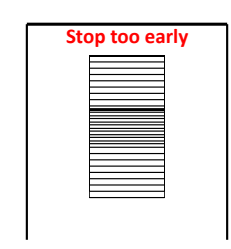

$\square$

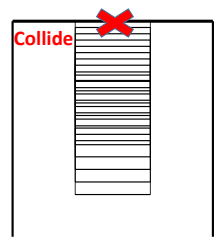

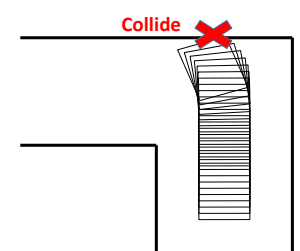

$\square$

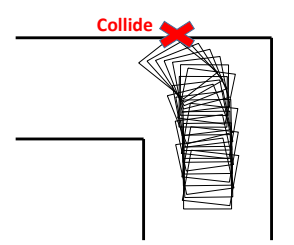

$\square$

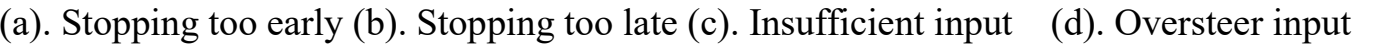

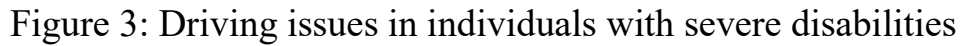

\title{
3. Shared control system using reinforcement learning
}

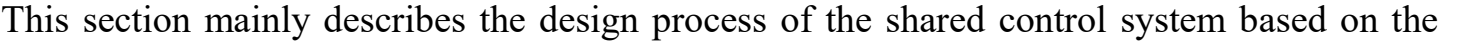

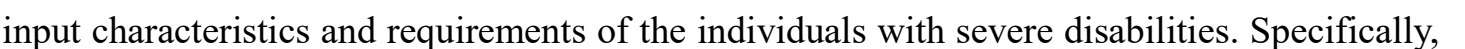

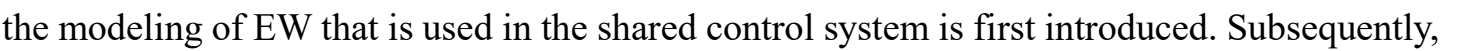
ए ए

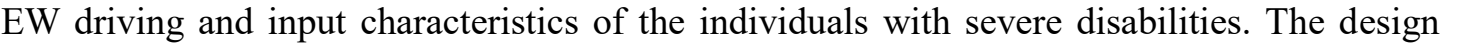

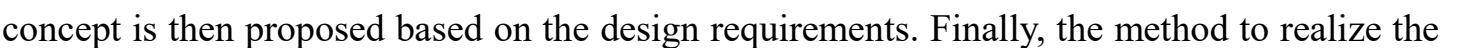

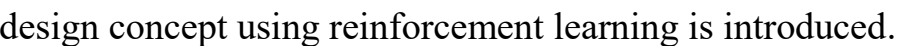

\subsection{Modeling an EW}

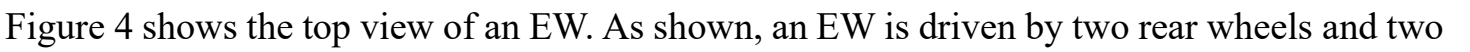

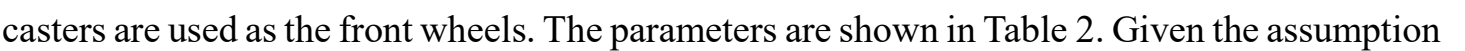
ए ए

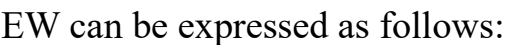

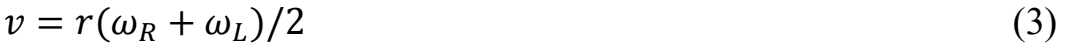

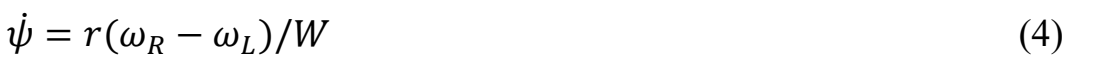

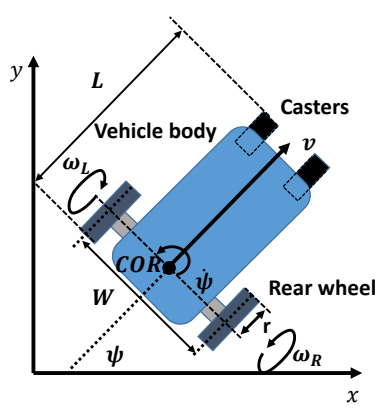

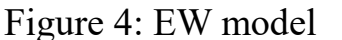

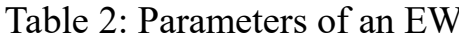

\begin{tabular}{|c|c|}
\hline 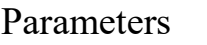 & 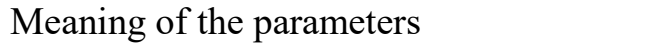 \\
\hline$\omega_{\mathrm{R}}, \omega_{L} \square$ & 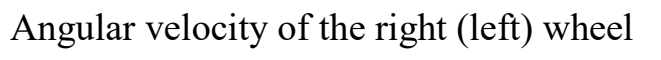 \\
\hline$r$ & 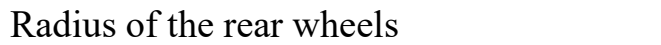 \\
\hline$W \square$ & 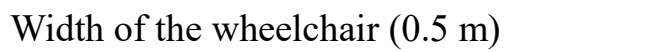 \\
\hline$L$ & 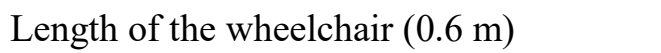 \\
\hline $\mathrm{v}, \dot{\psi} \square$ & 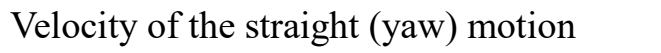 \\
\hline $\mathrm{x}, \mathrm{y} \square$ & 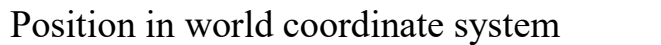 \\
\hline प्म०० & 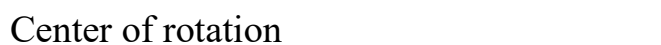 \\
\hline
\end{tabular}


Journal of Advanced Simulation in Science and Engineering

\subsection{Requirements of the shared control system}

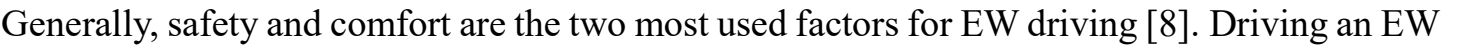

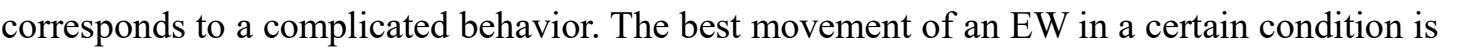

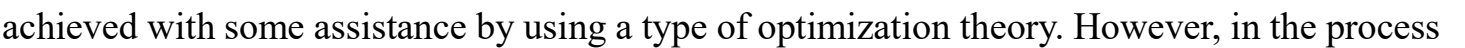

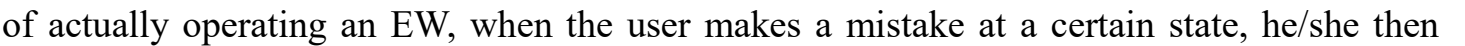

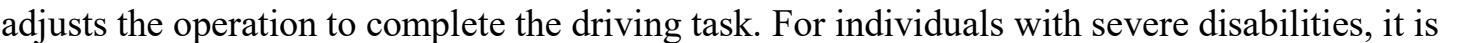

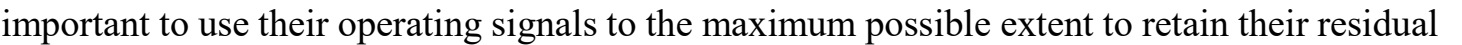
physical functions. Therefore, in order to completely utilize a user's operating ability, it is nec-

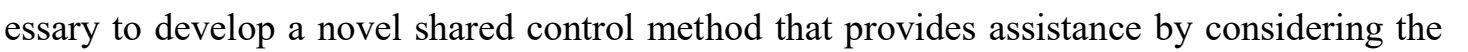

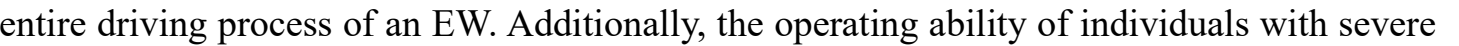

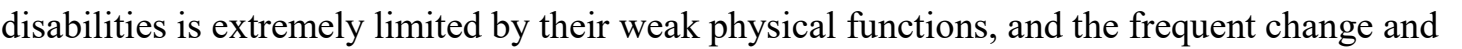

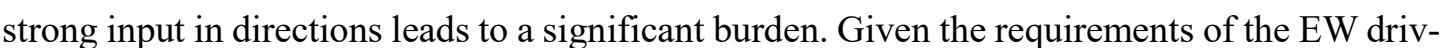
ing process, a user's physical condition, and input characteristics as discussed in Section 2, the

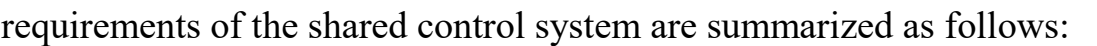

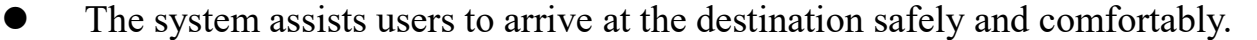

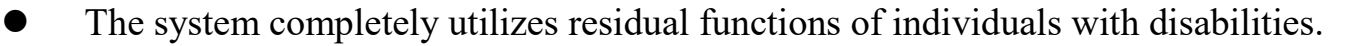

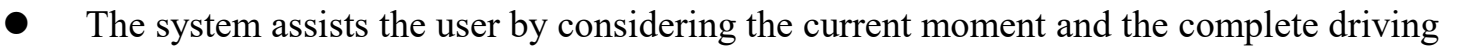
$\square\|\square\|\|\| \square$

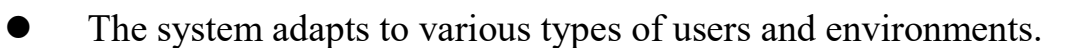

\subsection{Concept of the shared control system}

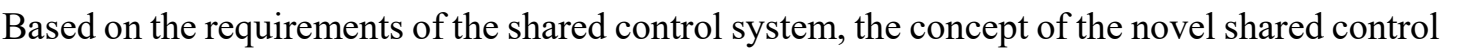

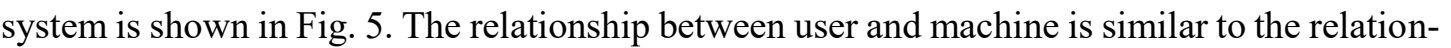

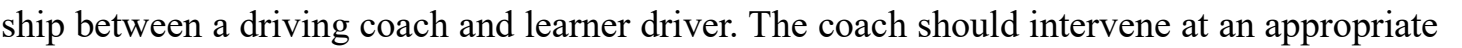

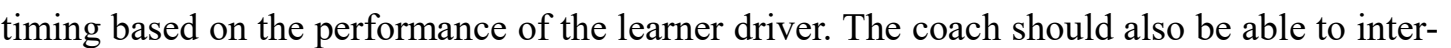

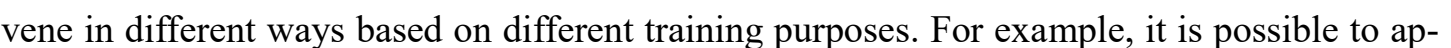

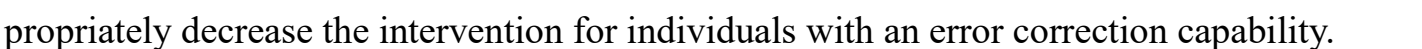

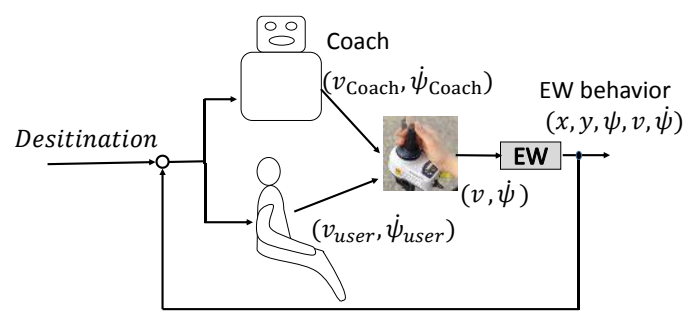

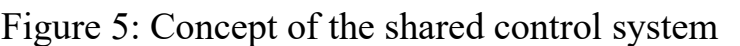


Journal of Advanced Simulation in Science and Engineering

\subsection{Framework of the proposed shared control system}

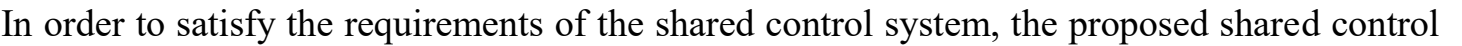

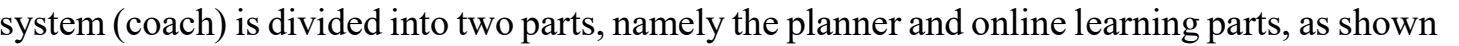

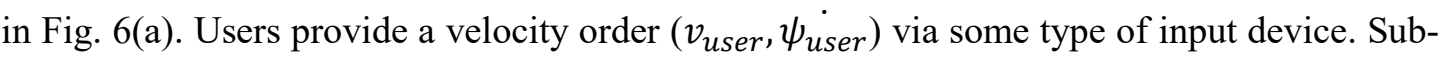

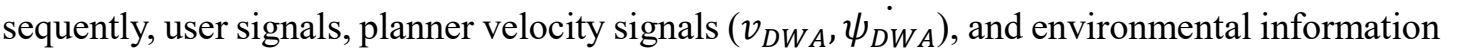

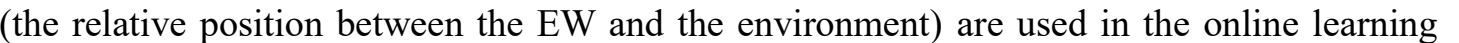

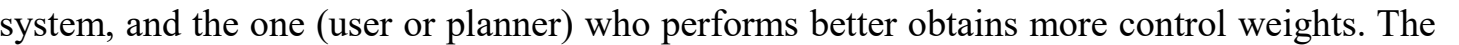

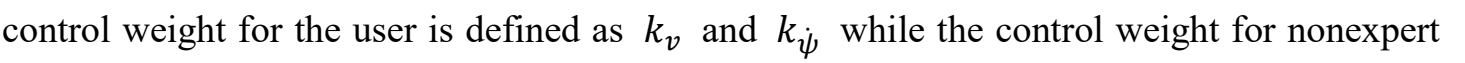

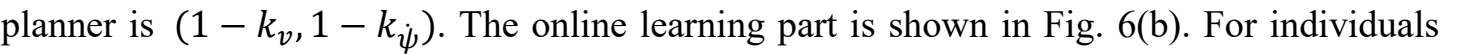

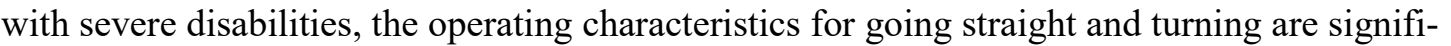

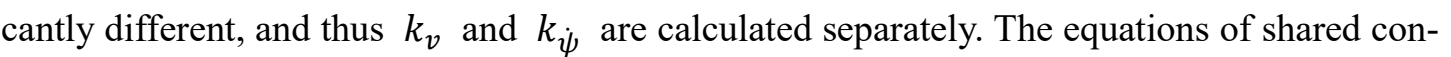

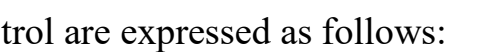

$$
\left\{\begin{array}{c}
v=k_{v} v_{u s e r}+\left(1-k_{v}\right) v_{D W A} \\
\dot{\psi}=k_{\dot{\psi}} \psi_{\text {user }}+\left(1-k_{\dot{\psi}}\right) \psi_{D W A}^{\dot{H}}
\end{array}\right.
$$

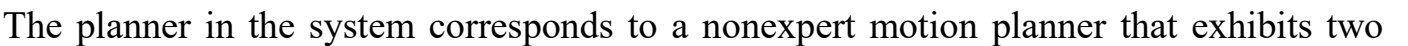

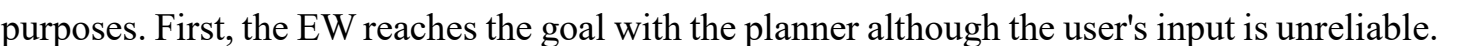

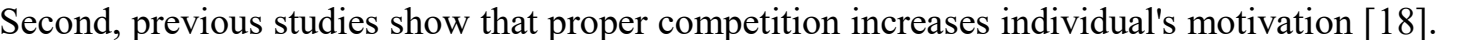

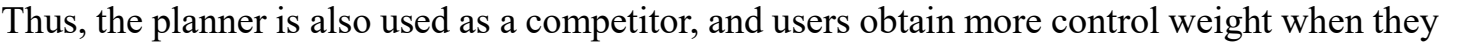

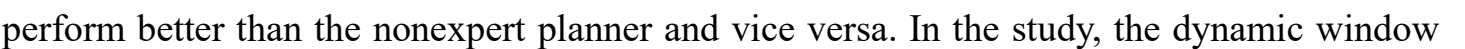

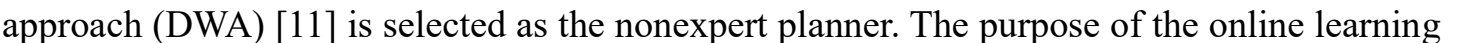

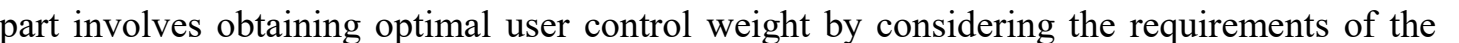

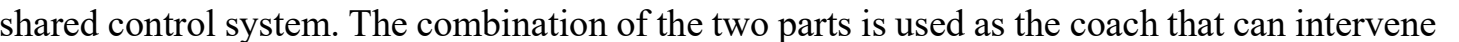

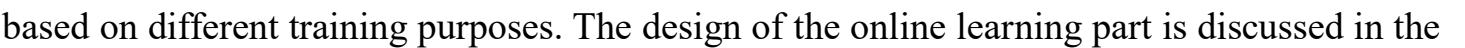

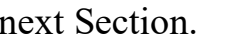

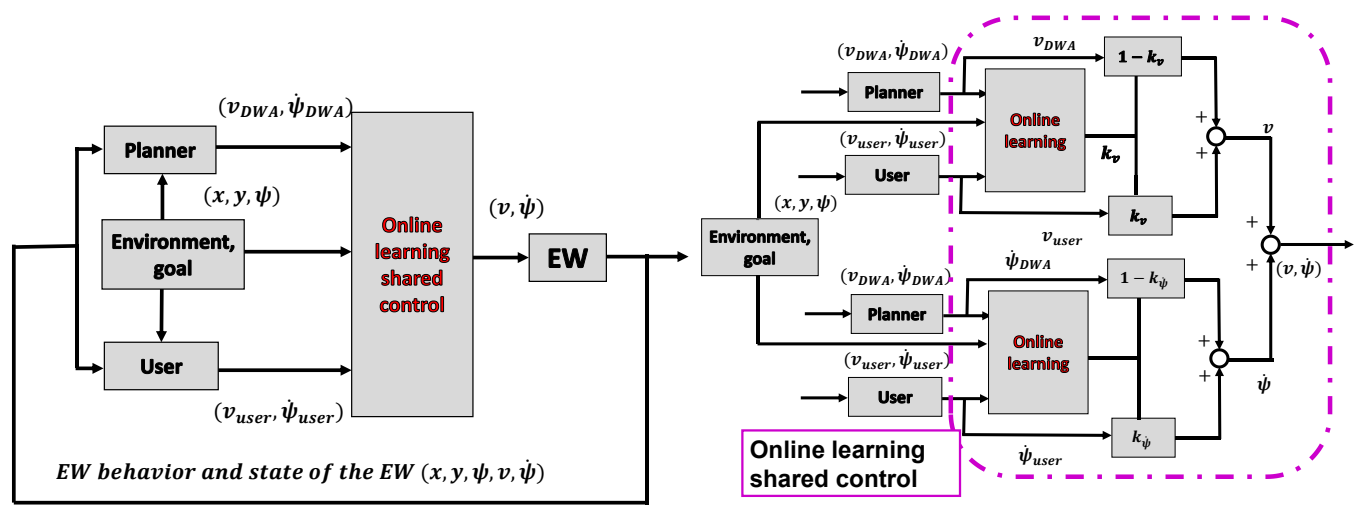

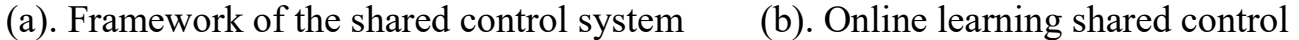


Journal of Advanced Simulation in Science and Engineering

\section{Shared controller design using reinforcement learning}

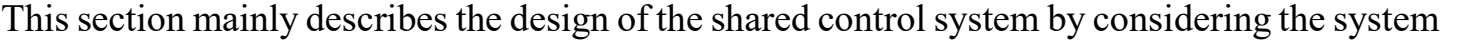

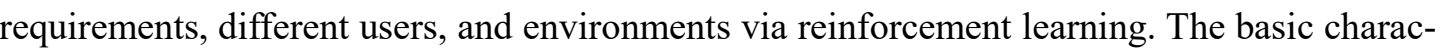

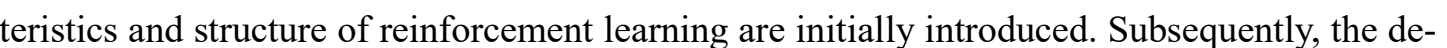

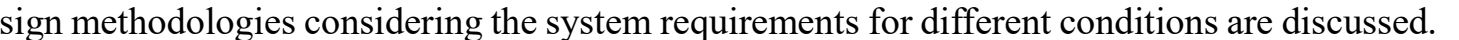

\subsection{Reinforcement learning}

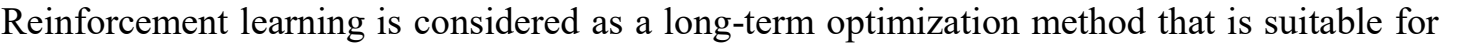

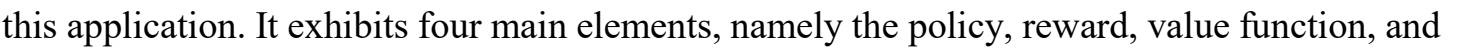

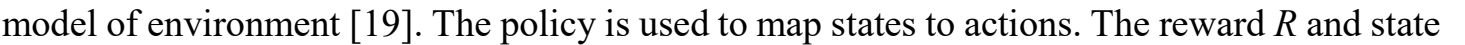

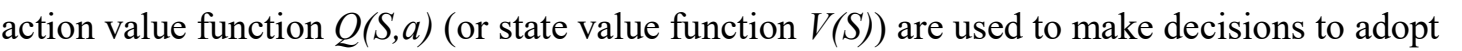

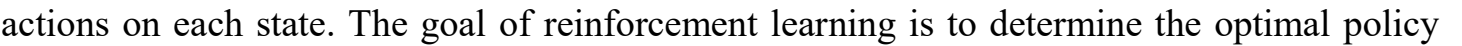

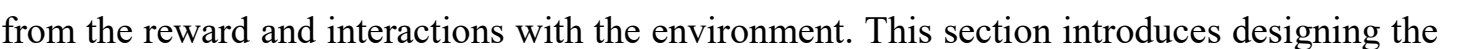

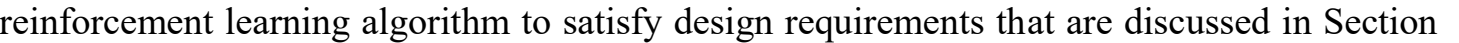
$\square$ पाए

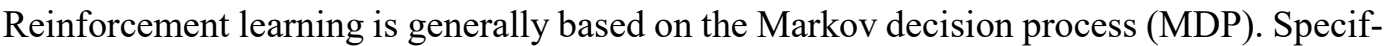

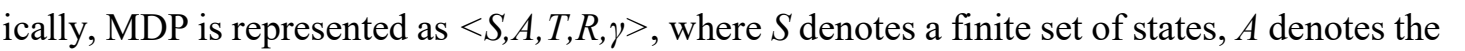

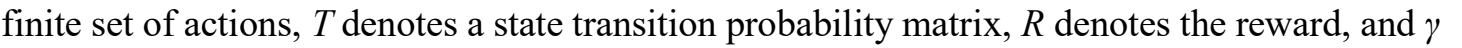

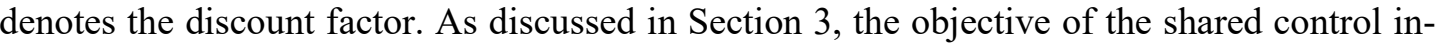

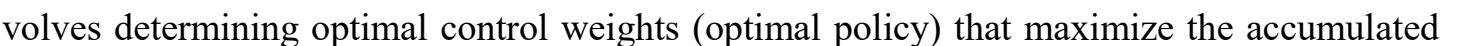

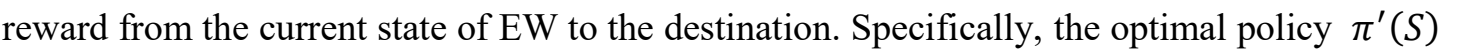

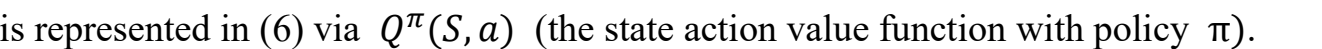

$$
\pi^{\prime}(S)=\underset{\mathrm{a} \in \mathrm{A}}{\operatorname{argmax}} Q^{\pi}(S, a)
$$

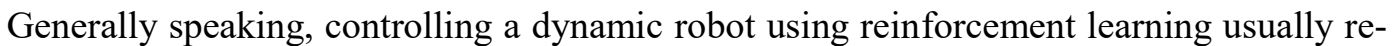

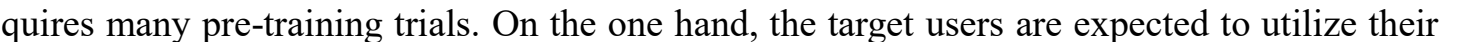

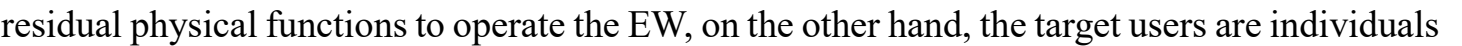

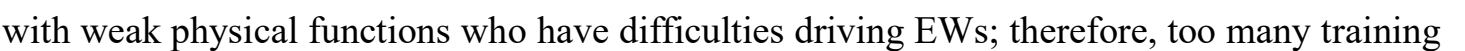
ए

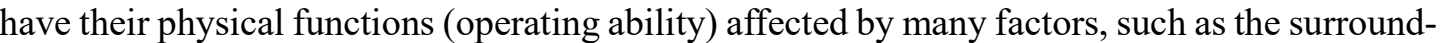

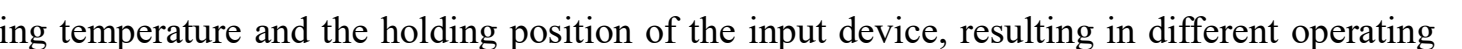

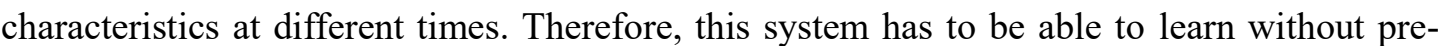

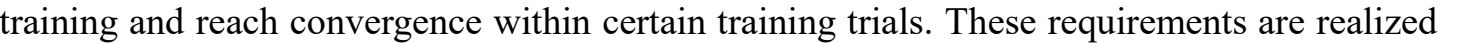

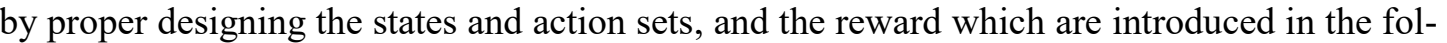

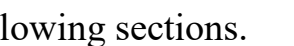




\section{Journal of Advanced Simulation in Science and Engineering}

\subsection{Design of discrete states}

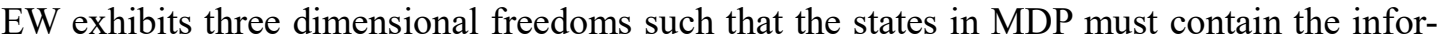

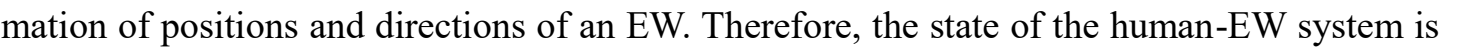

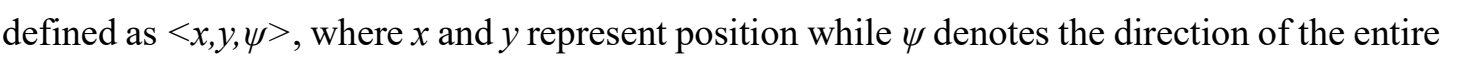

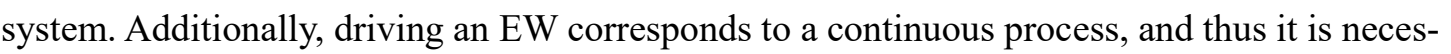

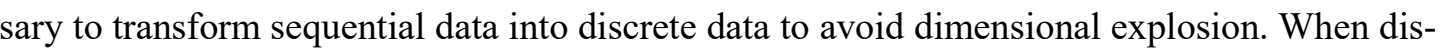

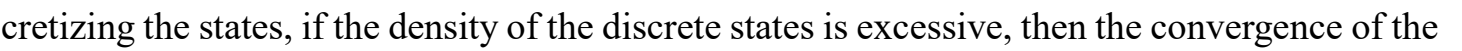

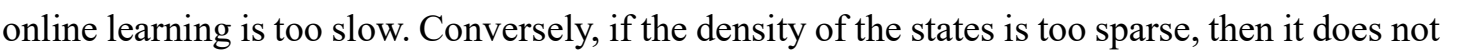

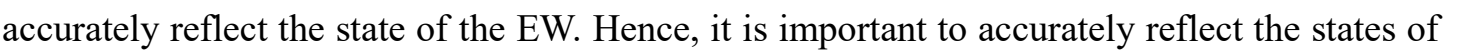

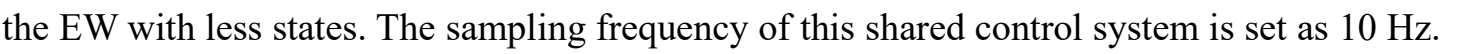

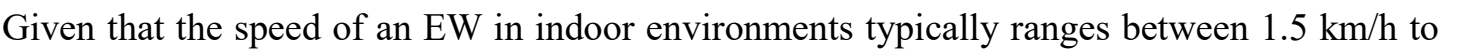

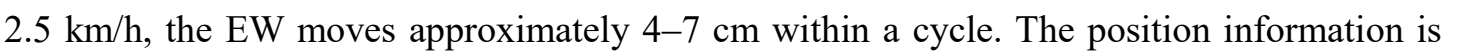

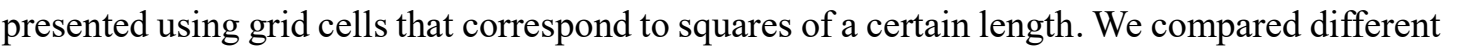

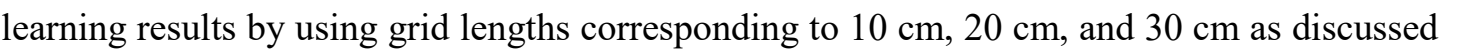

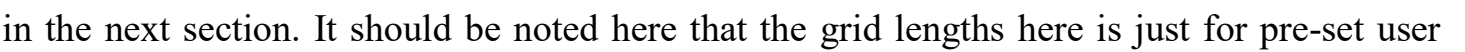

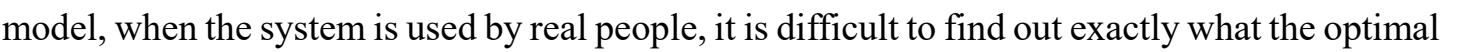

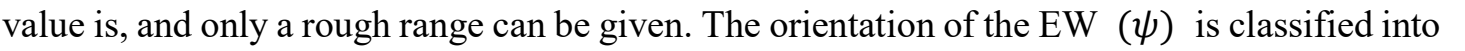

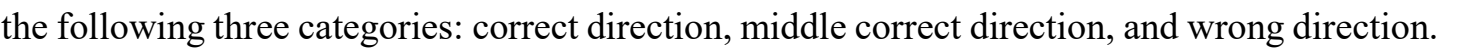

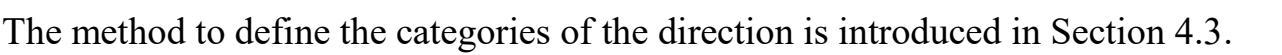

\subsection{Design of the reward function}

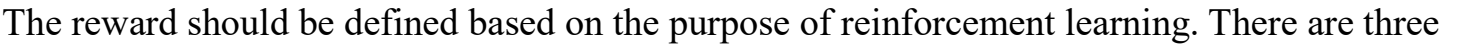

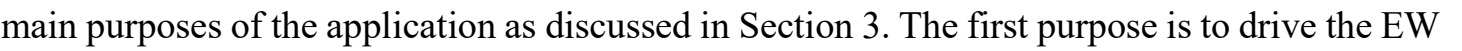

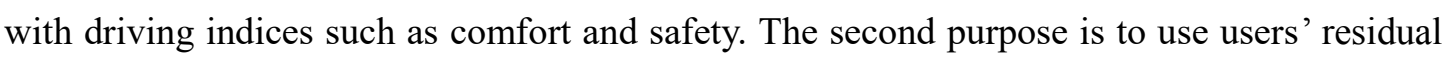

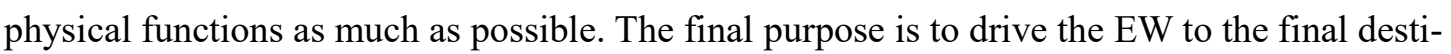

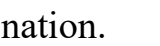

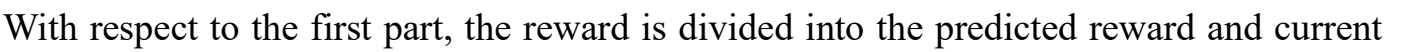

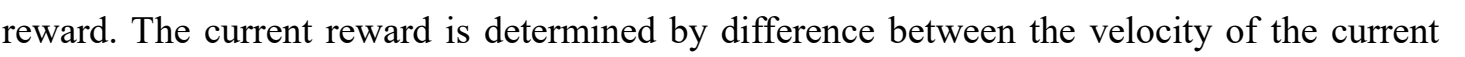

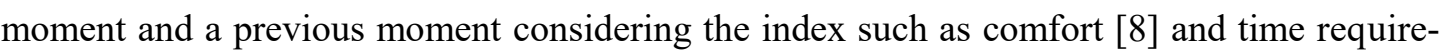

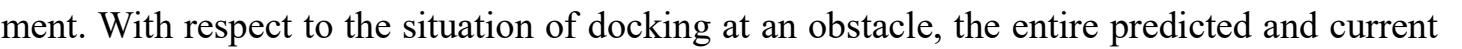

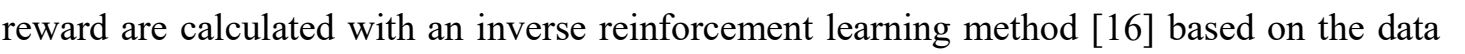

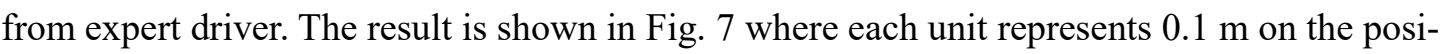

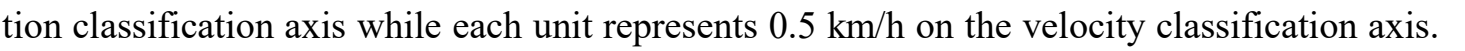

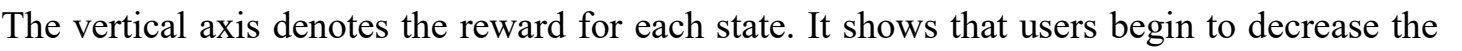

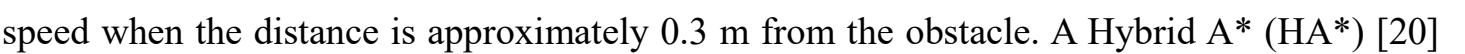




\section{Journal of Advanced Simulation in Science and Engineering}

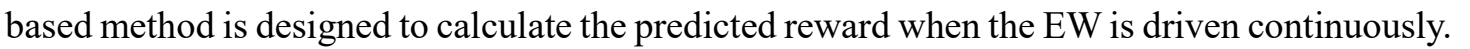

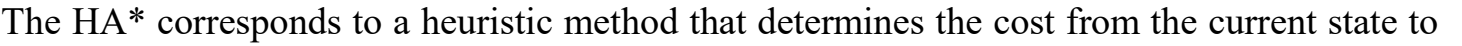
ए

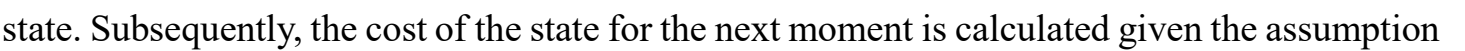

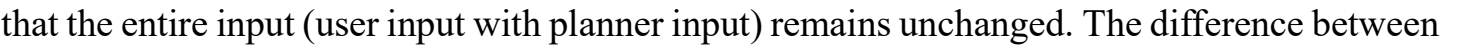

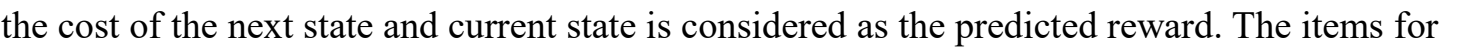

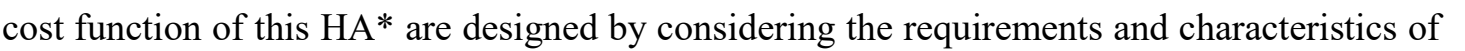

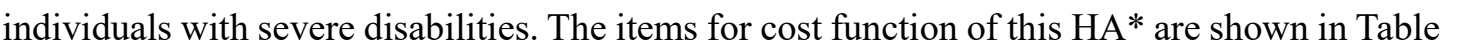

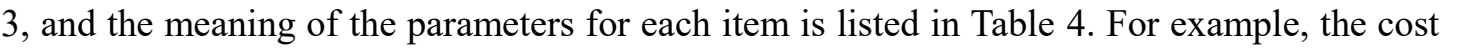

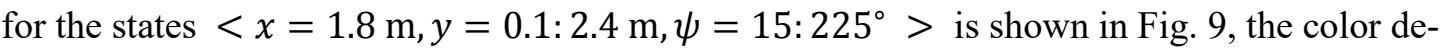

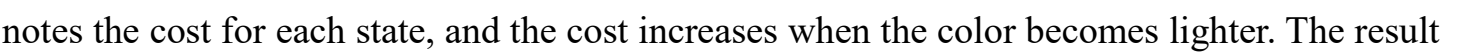

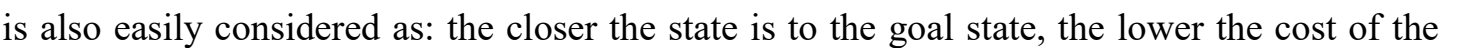

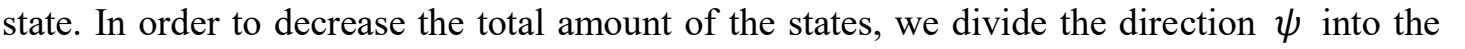

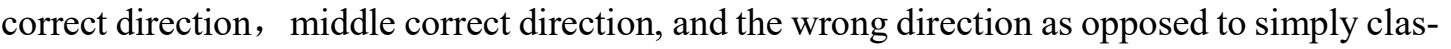

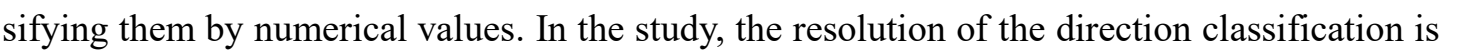

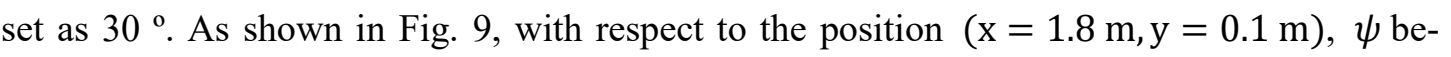

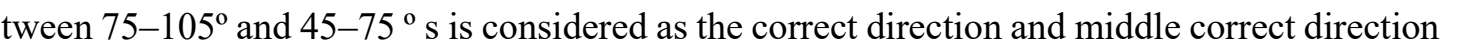

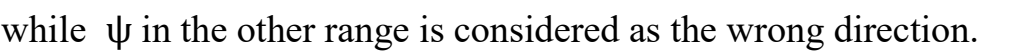

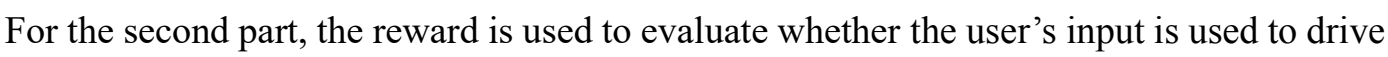

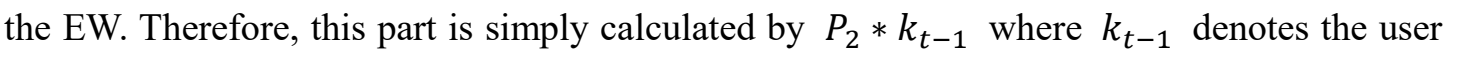

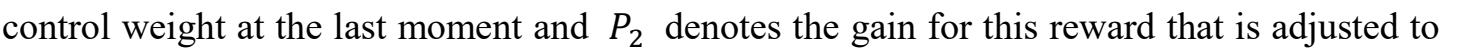

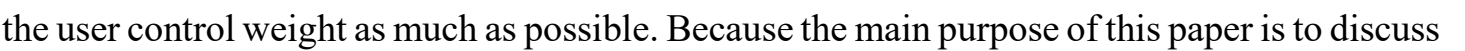

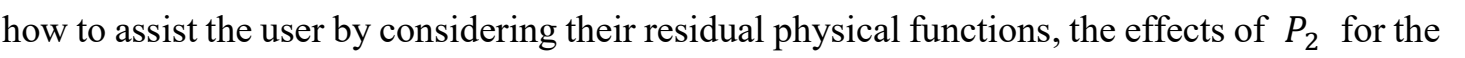

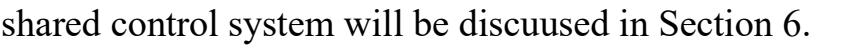

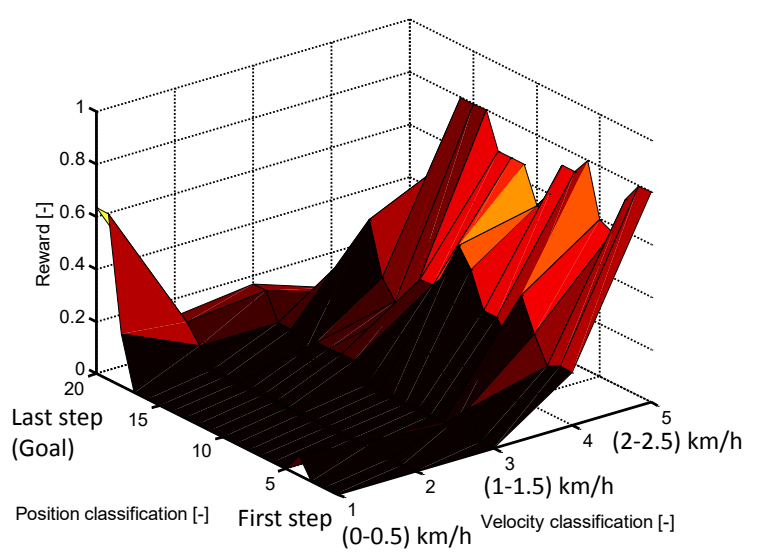

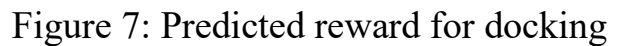


Journal of Advanced Simulation in Science and Engineering

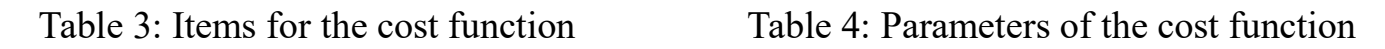

\begin{tabular}{|c|c|}
\hline 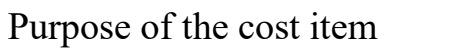 & 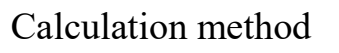 \\
\hline 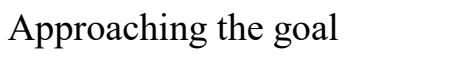 & $\mathrm{G}_{1} * \mathrm{HC} \square$ \\
\hline 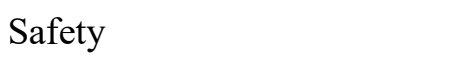 & $\mathrm{G}_{2} * d \square$ \\
\hline 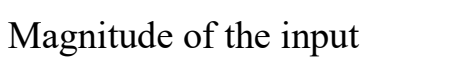 & $\mathrm{G}_{3} * \alpha \square$ \\
\hline 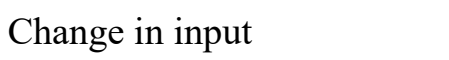 & $\mathrm{G}_{4} * \delta \alpha \square$ \\
\hline 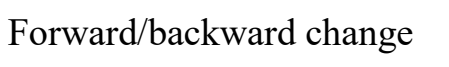 & $\mathrm{G}_{5} * S P \square$ \\
\hline$\square \square \square \square \square \square \square$ & $\mathrm{G}_{6} * B P \square$ \\
\hline
\end{tabular}

\begin{tabular}{|c|c|}
\hline$\square \square\|\square \square\| \square \square$ & 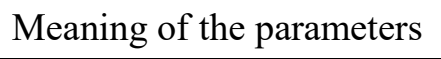 \\
\hline $\mathrm{G}_{\mathrm{n}} \square$ & 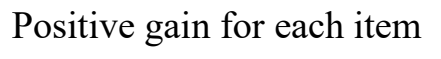 \\
\hline $\mathrm{HC} \square$ & 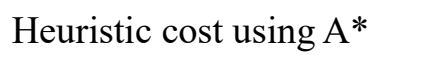 \\
\hline$d \square$ & 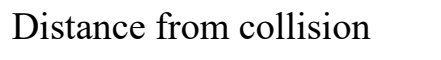 \\
\hline$\alpha(\delta \alpha) \square$ & 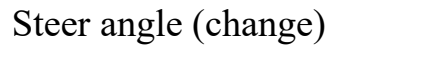 \\
\hline$S P \square$ & 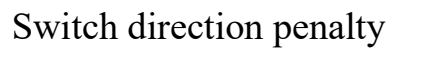 \\
\hline$B P \square$ & 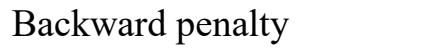 \\
\hline
\end{tabular}

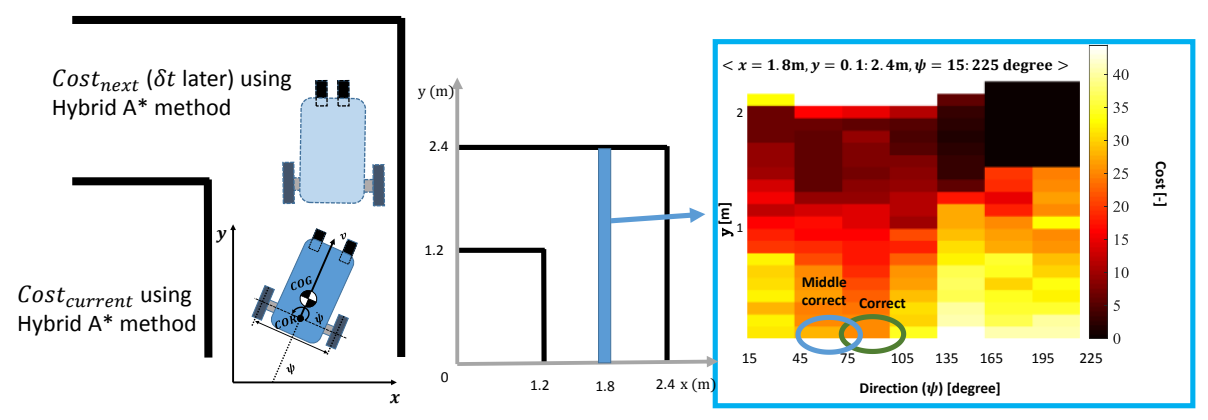

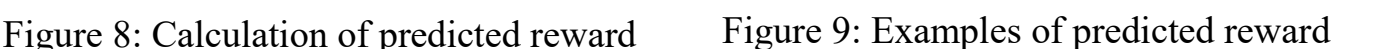

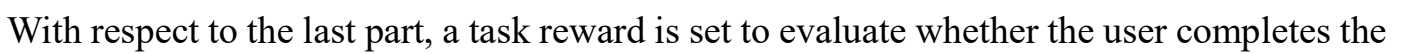

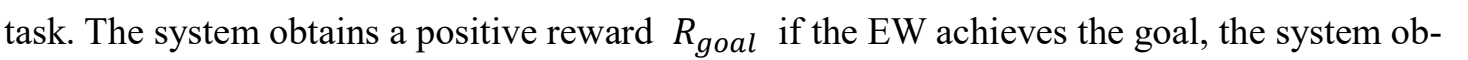

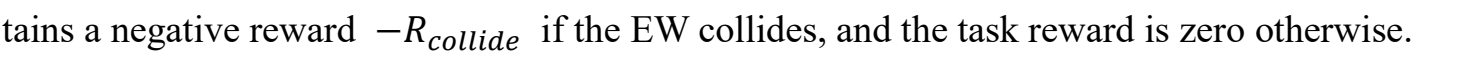

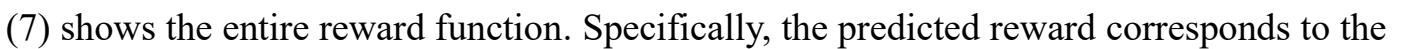

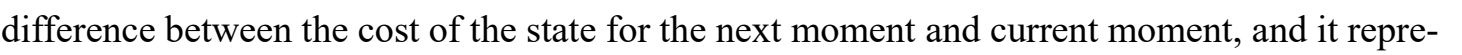

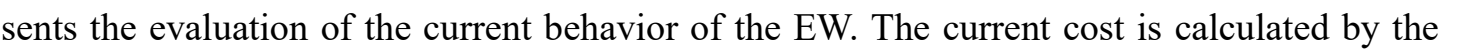

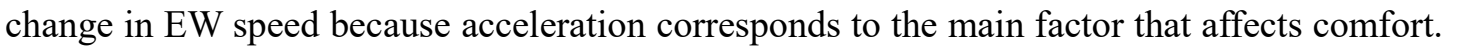

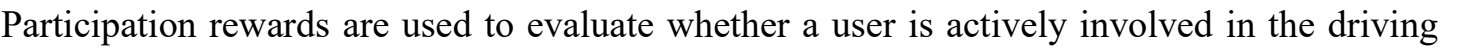

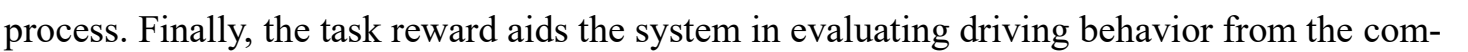

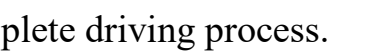

$$
\begin{aligned}
& \text { Reward }=\underbrace{\text { Cost }_{\text {current }}-\text { Cost }_{\text {next }}}_{\text {Predicted reward }}+\underbrace{\mathbf{P}_{1} * \operatorname{abs}\left(\mathrm{v}_{\mathrm{t}}-\mathrm{v}_{\mathrm{t}-1}\right)}_{\text {Current reward }} \\
& +\underbrace{\boldsymbol{P}_{2} * \boldsymbol{k}_{t-1}}_{\text {Participation reward }}+\underbrace{\boldsymbol{R}_{\text {goal }}-\boldsymbol{R}_{\text {collision }}}_{\text {Task reward }} \square\|\|\|\|\|\|\|\|\|\| \| \\
& \square \\
& \square \\
& \square
\end{aligned}
$$


Journal of Advanced Simulation in Science and Engineering

\subsection{Choice of the online reinforcement learning algorithm}

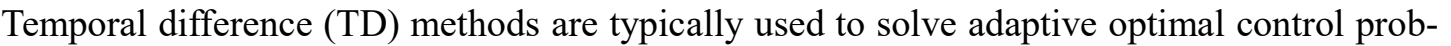

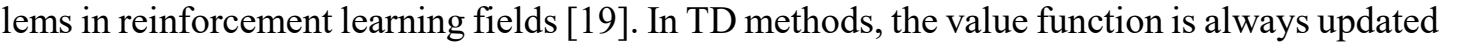

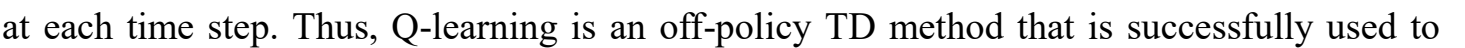

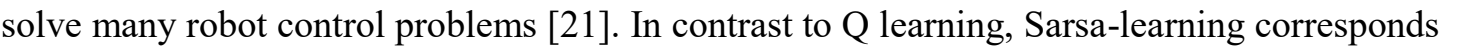

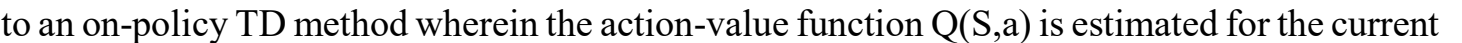

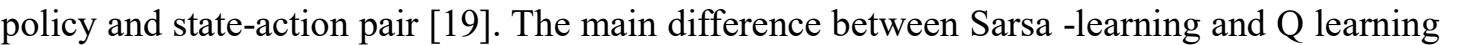
ए

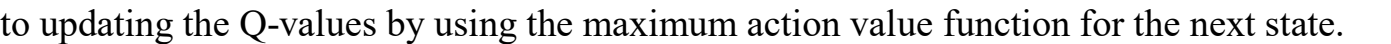

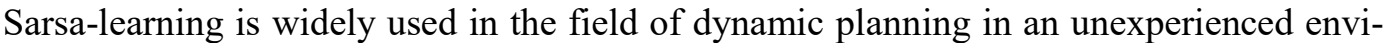

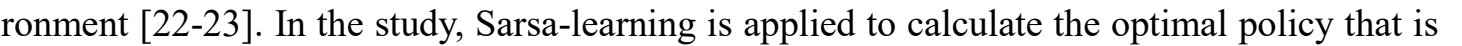

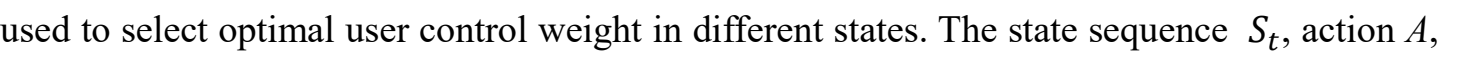

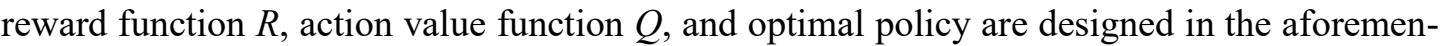

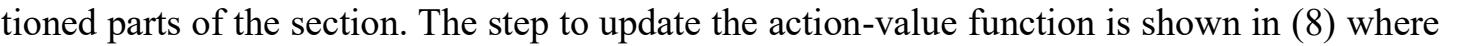

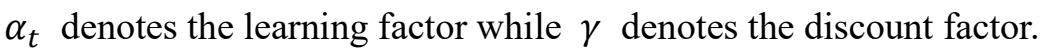

$$
\square Q\left(S_{t}, A_{t}\right)=Q\left(S_{t}, A_{t}\right)+\alpha_{t}\left[R_{t}+\gamma Q\left(S_{t+1}, A_{t+1}\right)-Q\left(S_{t}, A_{t}\right)\right] \square \amalg \Pi\|\Pi\| \Pi\|\| \amalg
$$

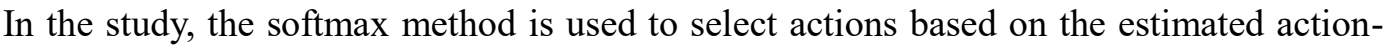

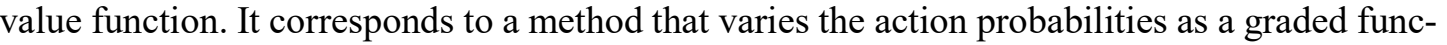

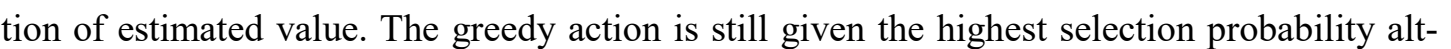

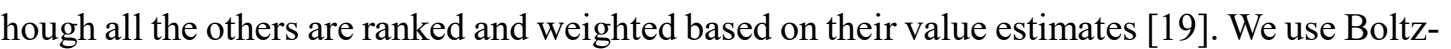

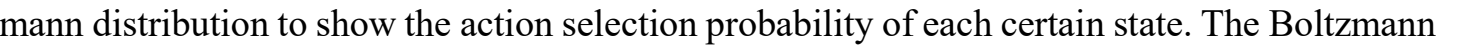

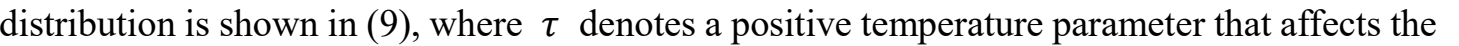

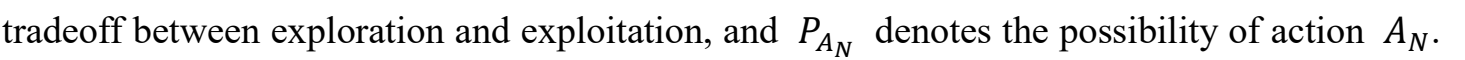

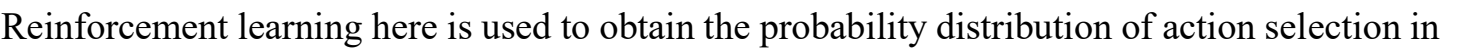

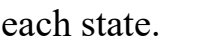

\|\|\|\|\|\|\|\|\|\|\|\|$\left\|\mathrm{p}_{\mathrm{A}_{\mathrm{N}}}=\frac{e^{Q\left(A_{N}\right) / \tau}}{\sum_{i=1}^{n} e^{Q\left(A_{i}\right) / \tau}} \square\right\|\|\|\|\|\|\|\|\|\|\|\|\|\|\|\|\|$

\subsection{Reinforcement learning based shared control algorithm}

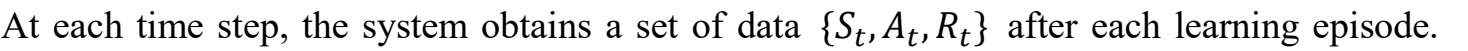

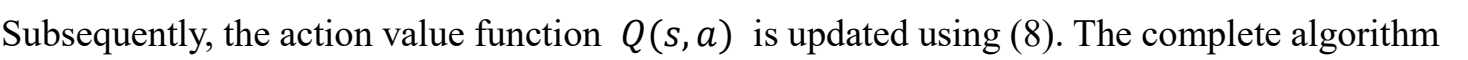

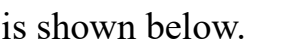

Algorithm 1. $t=0, \gamma=0.8, \alpha_{t}=0.05, R_{t}$ from reward function Algorithm 2. Recursively compute until the stop condition is met

Algorithm 2.1. Recursively compute until the goal is reached 
1. Obtain current state $S_{t}$

2. Decide action $A_{t}=\left(k_{v}, k_{\dot{\psi}}\right)$ by Sarsa-learning

3. Send $\left(k_{v}, k_{\dot{\psi}}\right)$ to the system, calculate output $(v, \dot{\psi})$

4. Send output $(v, \dot{\psi})$ to the $E W$

5. Calculate the next state $S_{t+1}$, Update $Q$ table, Load $R_{t} \square$

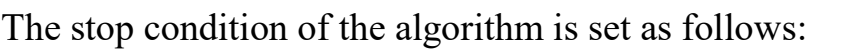

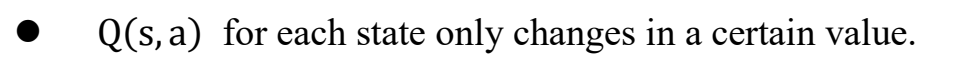

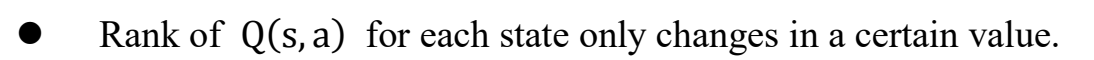

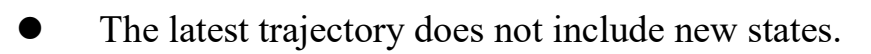

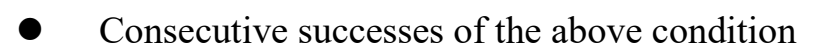

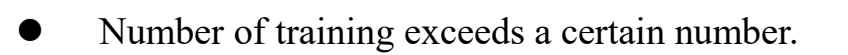

\section{Simulation studies}

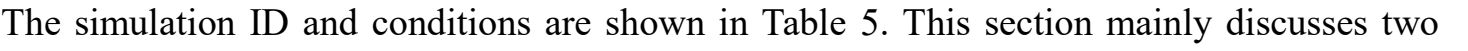

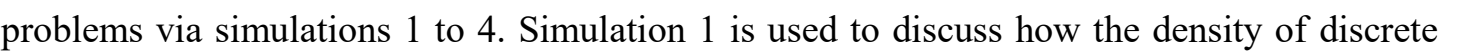

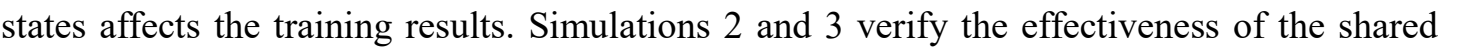

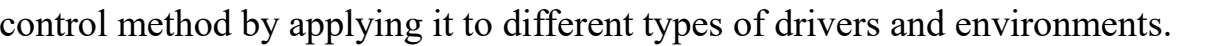

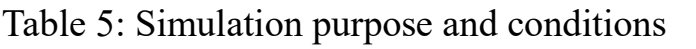

\begin{tabular}{|c|c|c|}
\hline 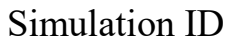 & $\square \square \square \square \| \square \square \square \square$ & $\square \square\|\|\|\square \square \square\|$ \\
\hline$\square$ & 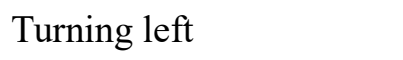 & 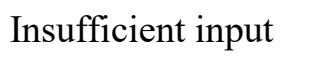 \\
\hline$\square$ & 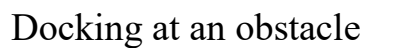 & 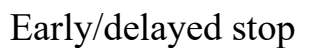 \\
\hline$\square$ & 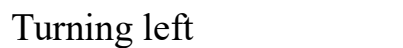 & 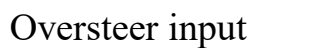 \\
\hline
\end{tabular}

\subsection{Designing the density of discrete states}

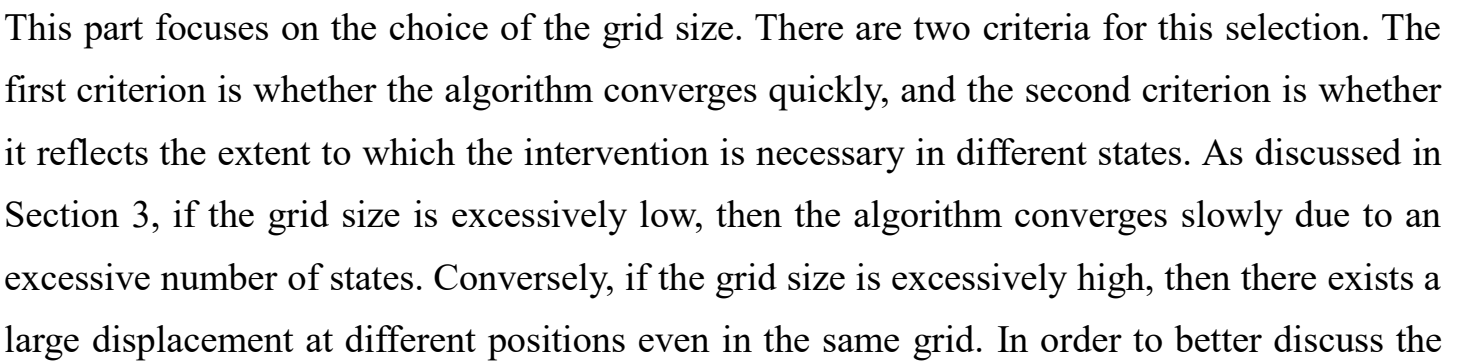




\section{Journal of Advanced Simulation in Science and Engineering}

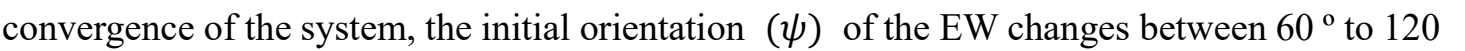

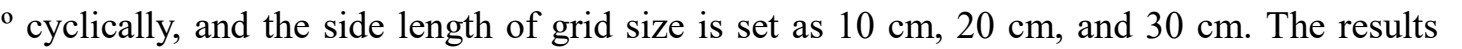

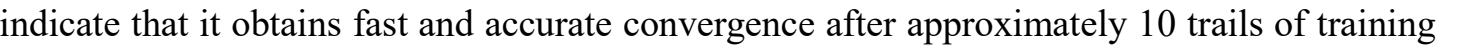

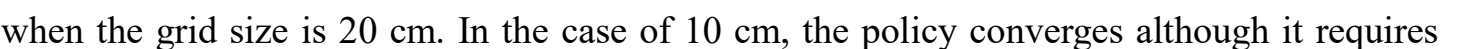

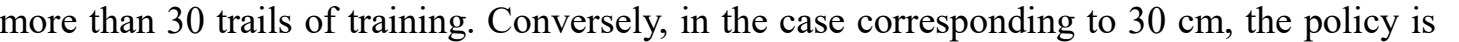

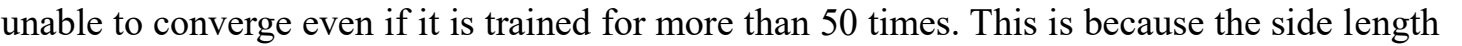

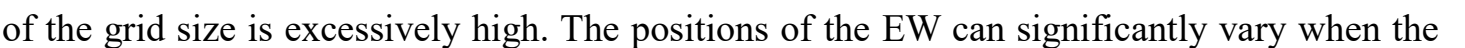

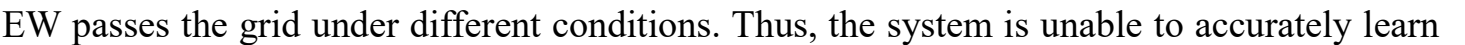

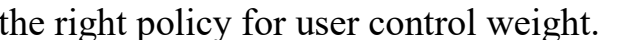

\subsection{Verification of the effectiveness in various environments}

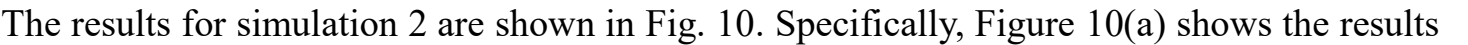

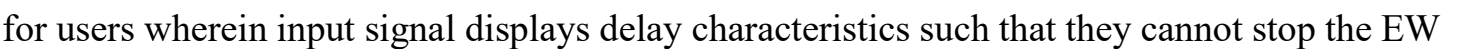

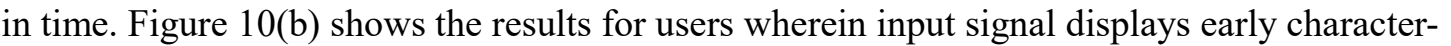

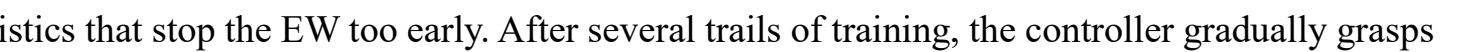

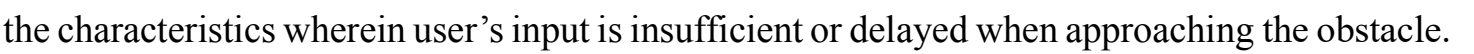

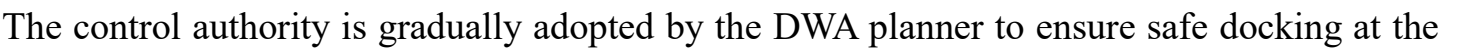

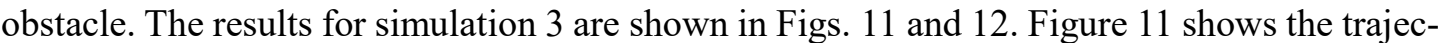

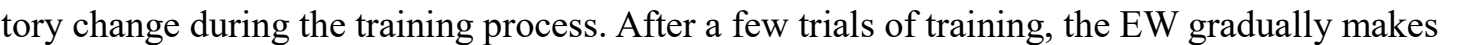

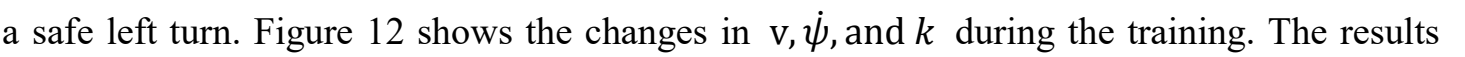

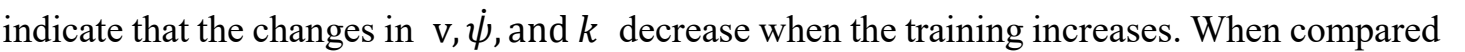

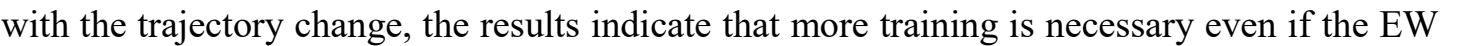

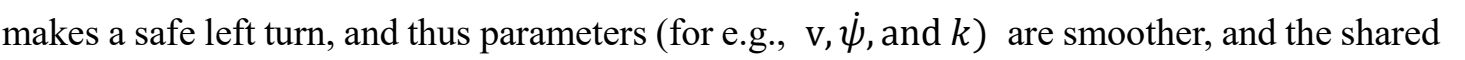

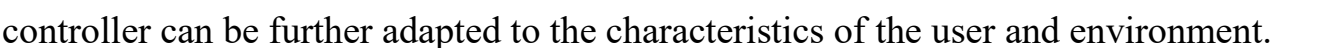
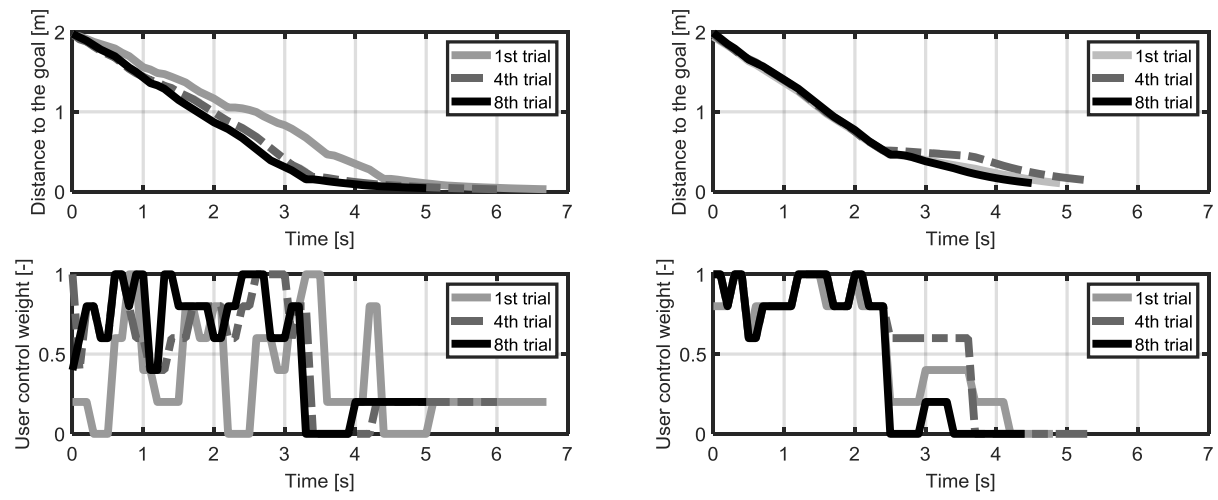

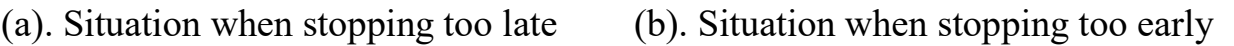



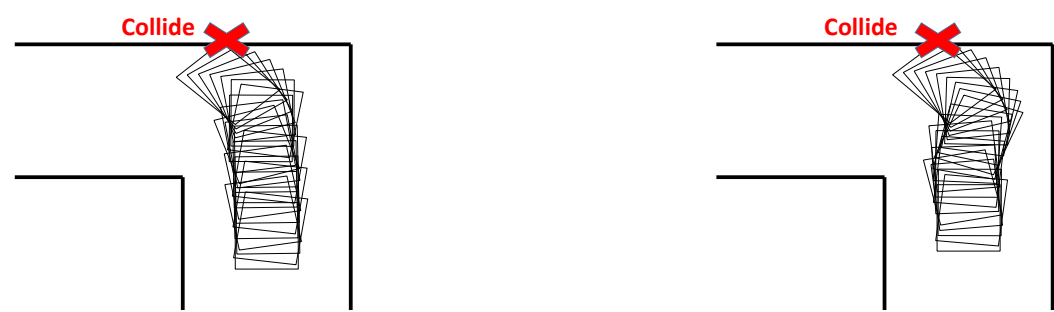

$\square|||||||| \mid \square$

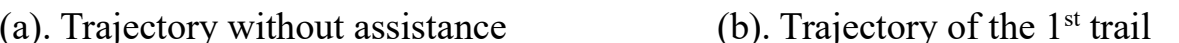
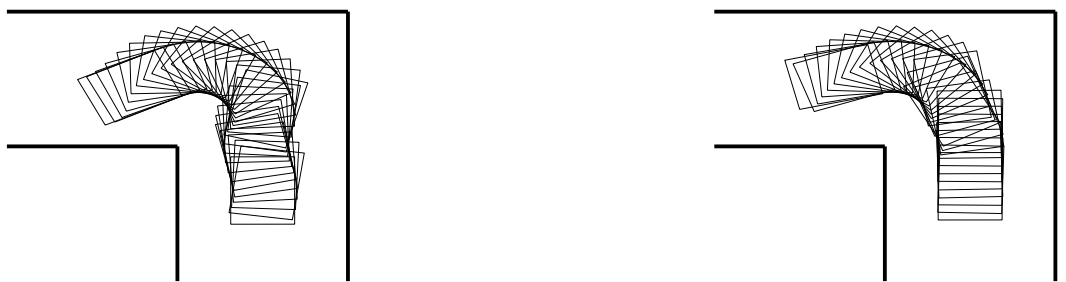

$\square\|\|\|\|\|\| \square$

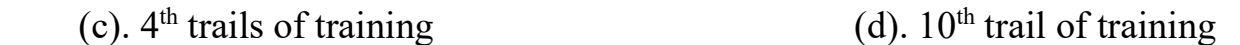

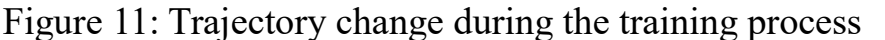
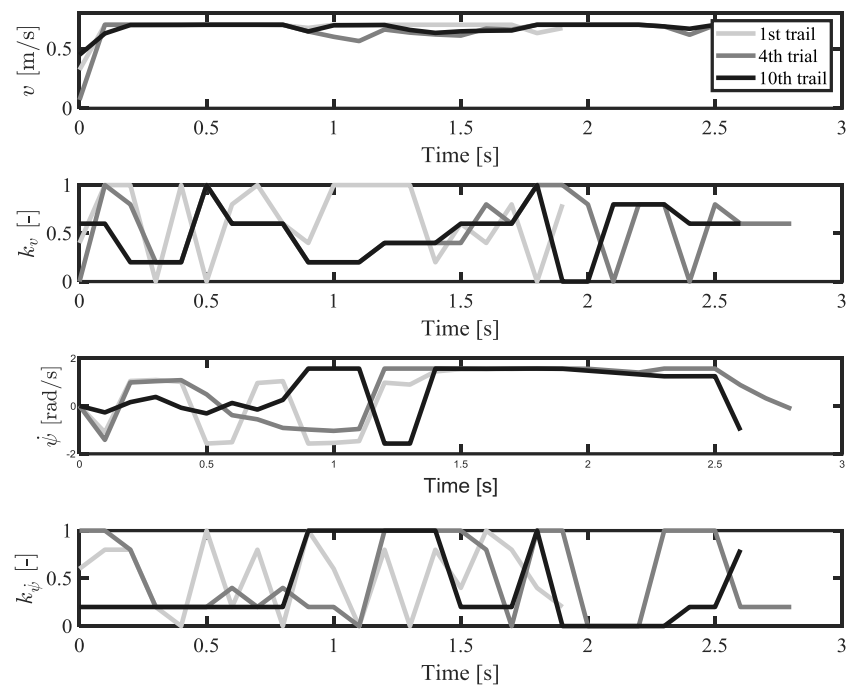

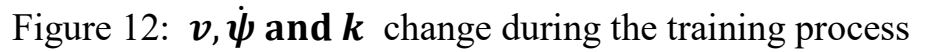

\section{Discussion}

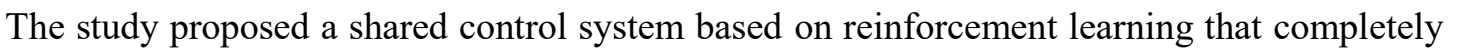

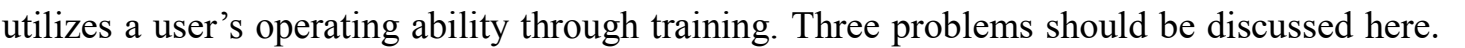


Journal of Advanced Simulation in Science and Engineering

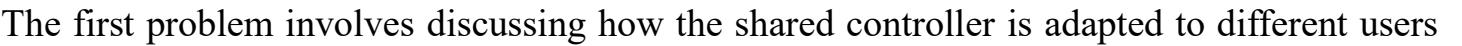

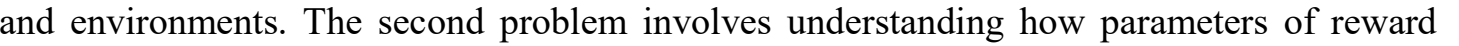

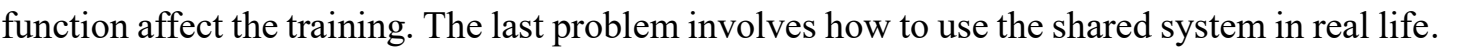

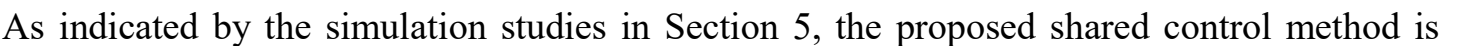

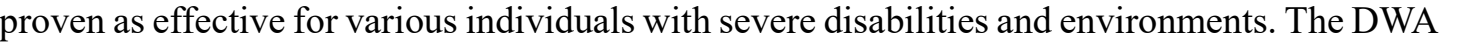
can be considered as a minimum guarantee wherein if the user's input is not sufficient to oper-

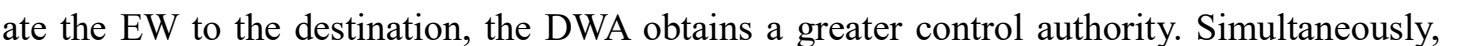

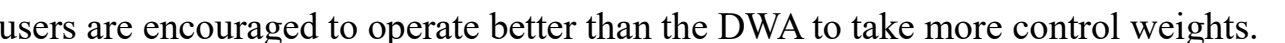

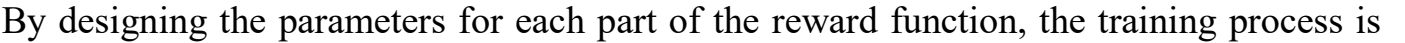

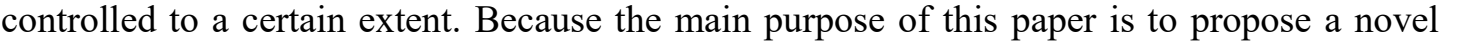
पण

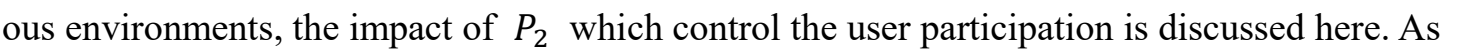

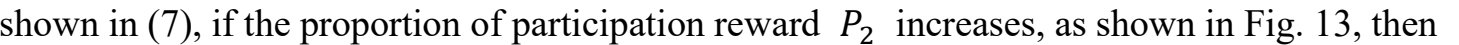
the system attempts to increase the user's participation. However, user control weight $\square \square \square \square \square \square$

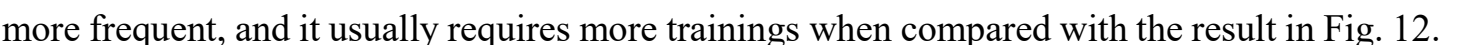

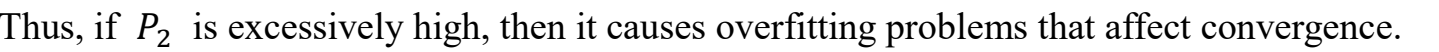

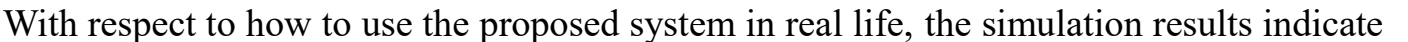

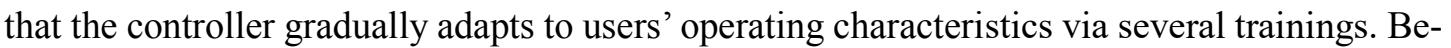

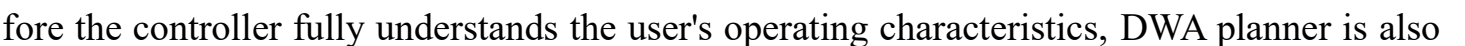

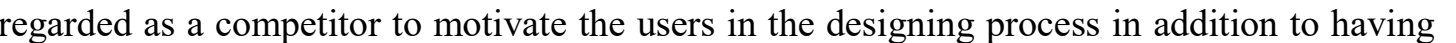

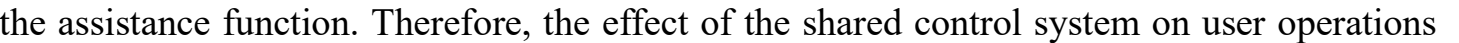

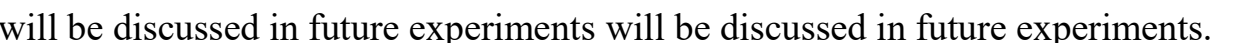

\section{Conclusions and future work}

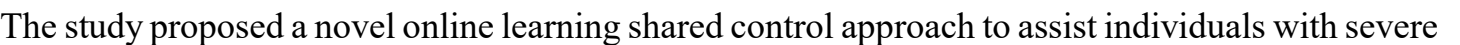

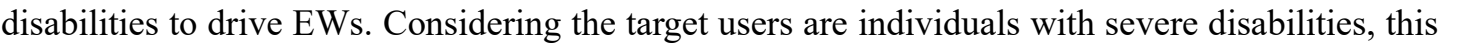

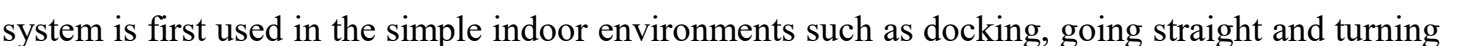

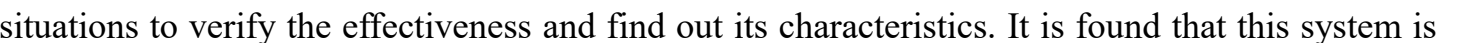

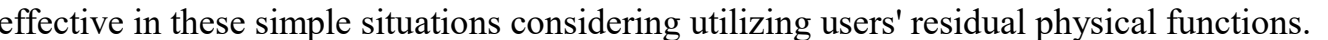

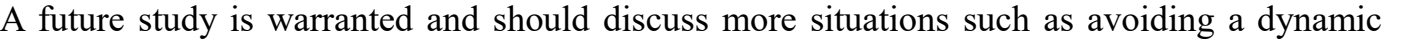

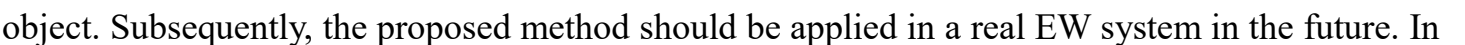

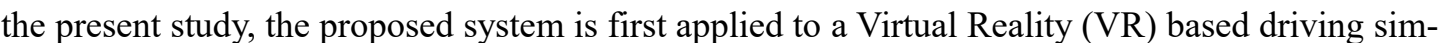

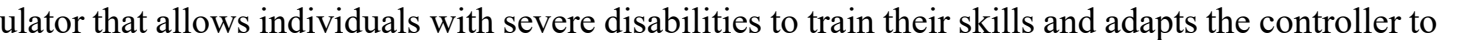
users' characteristics. The trained controller is then used for real EW driving. $\square$ 

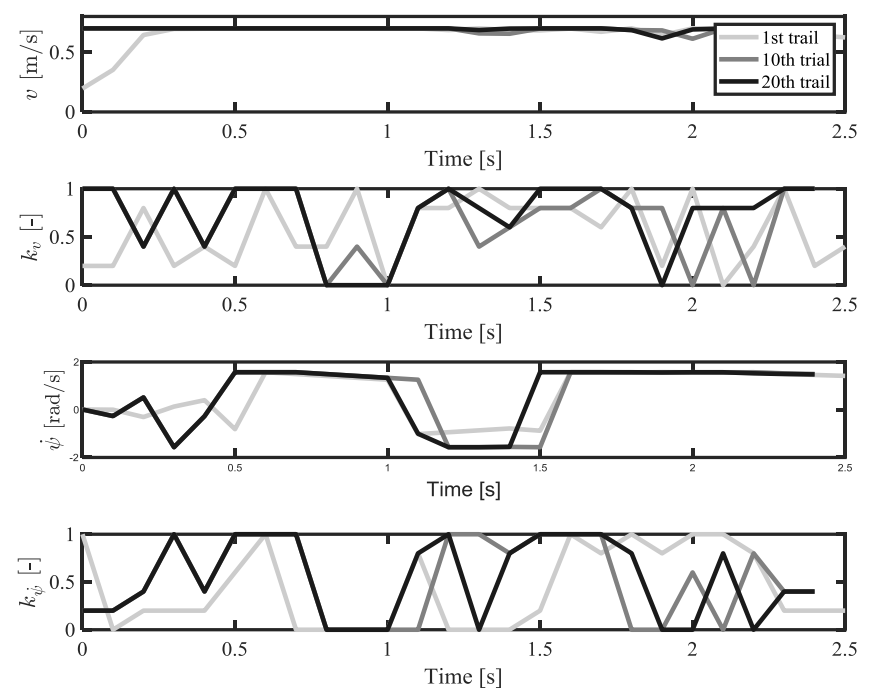

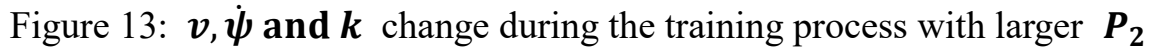

\section{References}

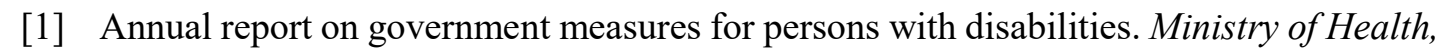

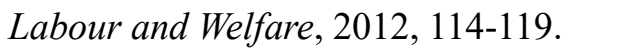

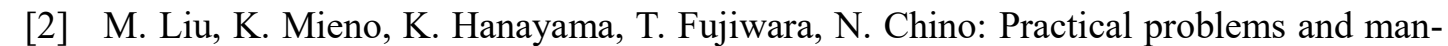

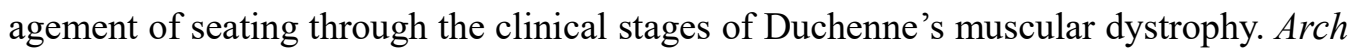

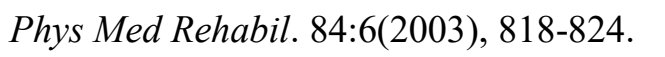

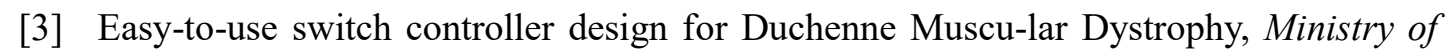

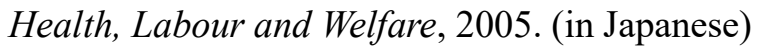

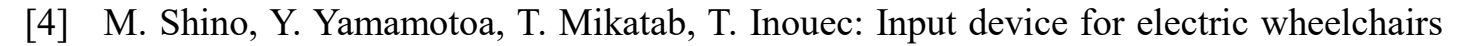

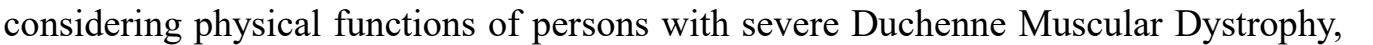

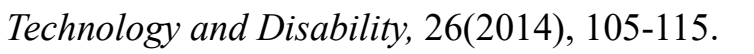

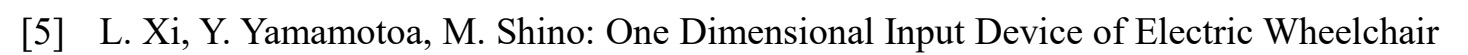

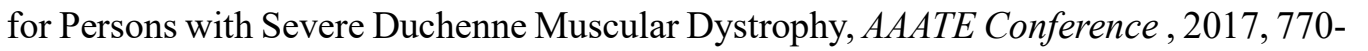
$\square 10$

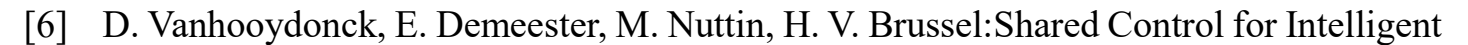

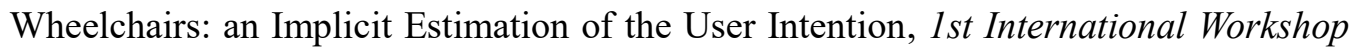

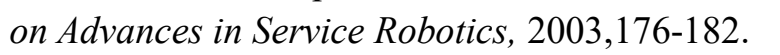

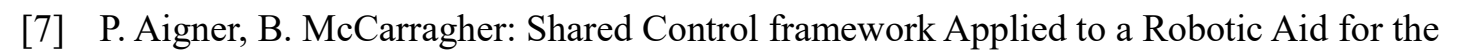
$\square \square \square \square$ IEEE Control System $\square 1 \square \square \square-\square \square$

$\square \square$
$\square \square \square$

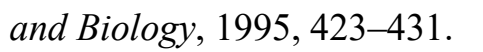

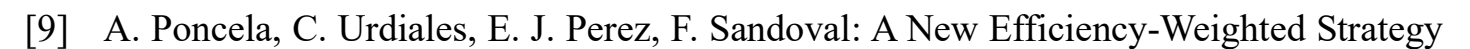


Journal of Advanced Simulation in Science and Engineering

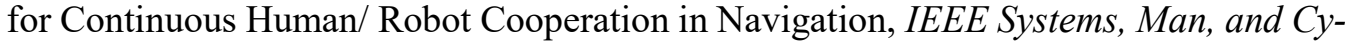
bernetics Society

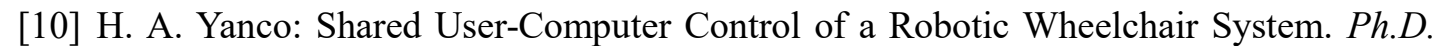

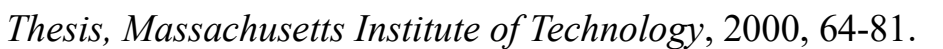

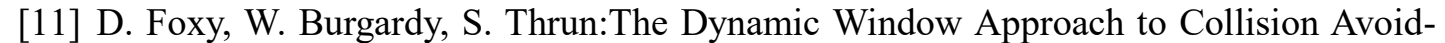

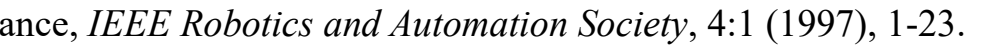

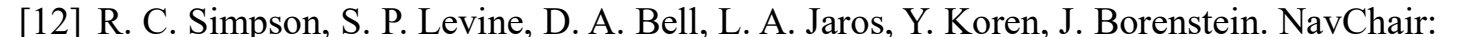

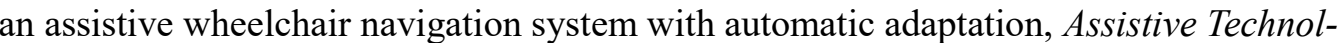

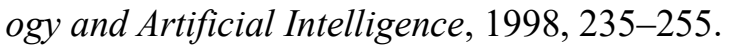

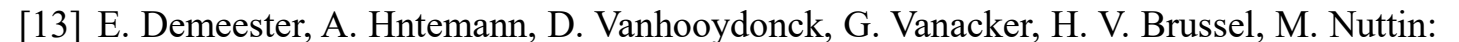

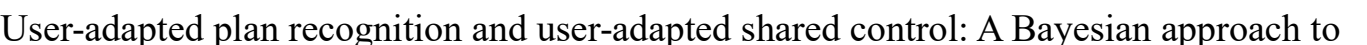

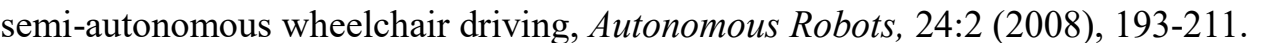

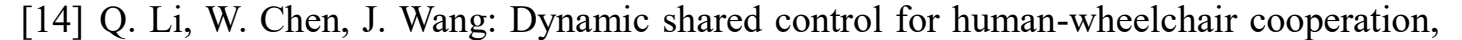

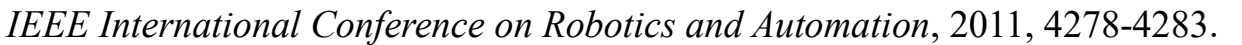

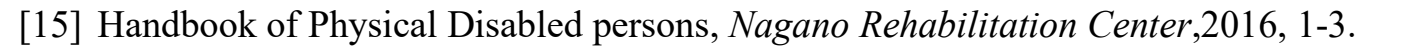

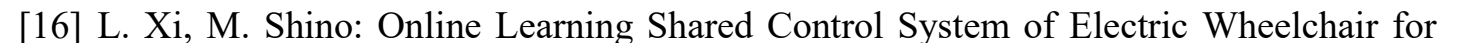

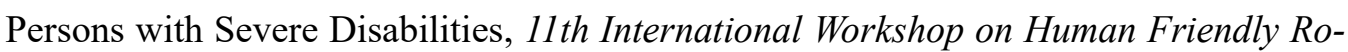

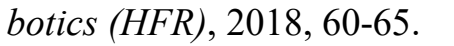

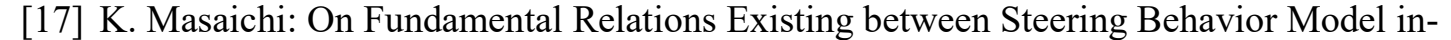

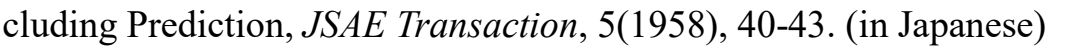

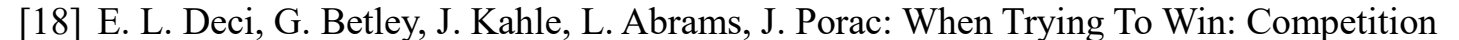

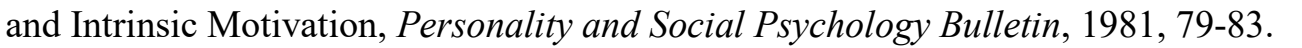

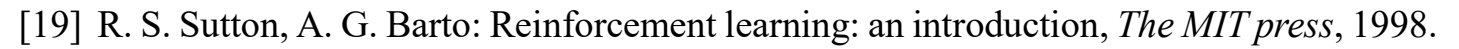

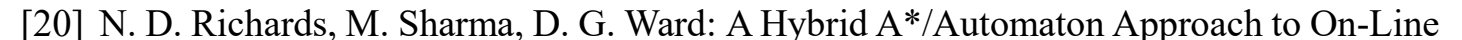

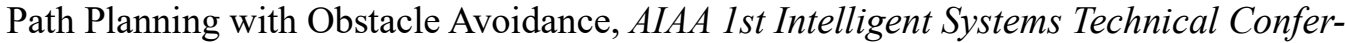

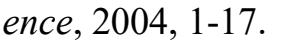

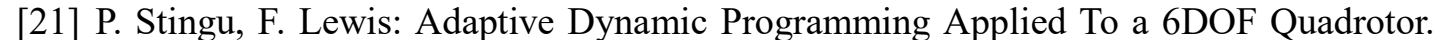
Computational modeling and simulation of intellect: Current state and future perspec-

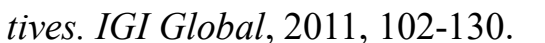

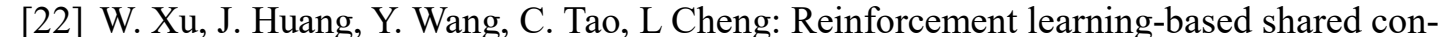

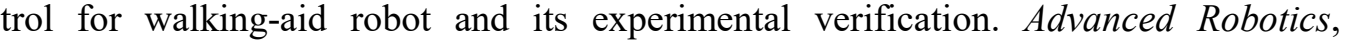

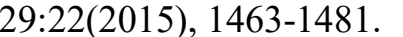

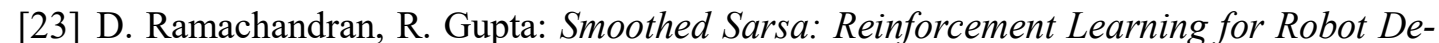
livery Tasks $\square 2009$ IEEE International Conference on Robotics and Automation $\square \square \square \mid \square$ $\square|\square|\|1\| \square$ 